\title{
Observability of switching structured linear systems with unknown input. A graph-theoretic approach
}

\author{
T. Boukhobza and F. Hamelin \\ Centre de Recherche en Automatique de Nancy (CRAN), Nancy-University, CNRS UMR 7039 \\ email: taha.boukhobza@cran.uhp-nancy.fr
}

\begin{abstract}
This paper deals with the observability of the discrete mode, the internal state and the input of switching structured linear systems with unknown input. The proposed method, based on a graph-theoretic approach, assumes only the knowledge of the system's structure. We express, in graphical terms, necessary and sufficient conditions for the generic observability of the discrete mode, the continuous internal state and the input of a switching structured linear system. These conditions can be implemented by classical graph-theory algorithms based on finding particular paths and cycles in a digraph.
\end{abstract}

Key words: Switching linear systems, Mode observability, State and input observability, Graph theory.

\section{Introduction}

Hybrid systems, combining event-driven and time-driven dynamics, have received growing attention in the control community as they can be used to describe a wide range of physical and engineering systems. For such systems, the general problem of estimating the mode, the internal state and the unknown input is of great interest in many control areas. In the context of switching continuous time linear systems, studies presented in (Babaali and Pappas, 2005; De Santis et al., 2006; De Santis et al., 2009; Vidal et al., 2003) are among the most significant works which deal with observability. They characterize completely the observability of the continuous internal state and/or the discrete mode variable, which is assumed to be unknown and arbitrary. Other definitions of observability for other kinds of hybrid systems are also provided in the literature. For instance, (Bemporad et al., 2000; Collins and van Schuppen, 2004; Chaib et al., 2005) study the observability of hybrid linear systems, where the discrete mode depends on the state trajectory or are associated to discrete outputs. From the point of view of the kind of systems and observability definitions, our paper is close to (Babaali and Pappas, 2005; De Santis et al., 2006; Vidal et al., 2003; De Santis et al., 2009). But, our proposed method and the obtained results present two main originalities. On the one hand, few works deal with the observability of hybrid systems with unknown input. In fact, there exist some works ((Pina and Botto, 2006) and the references therein), which aim at designing unknown input observers for SLS. (Vu and Liberzon, 2006) addresses the problem of recovering the discrete mode variable and the input given an output and an initial state. Nevertheless, these works do not study state and input observability. On the other hand, in most cases, observability analysis for SLS deal with algebraic and geometric tools and so requires the exact knowledge of the state space matrices characterizing the systems' model. In many modeling problems or during conception stage, these matrices have a number of fixed zero entries determined by the physical laws while the remaining entries are not precisely known. Thus, to study the structural properties, we can consider models where the fixed ze- ros are conserved while the non-zero entries are replaced by free parameters. Many interesting works on these models, called structured models, are related to the graph-theoretic approach to analyse efficiently structural properties (Dion et al., 2003). In this context, our aim is to use such approach to characterize the observability of the discrete mode variable, the internal state and the input for switching structured linear systems (SSLS).

The paper is organised as follows: after Section 2, which is devoted to the problem formulation, some definitions related to the graph-theoretic approach are given in Section 3. The main results are enounced in Section 4 before a conclusion. 2 Problem statement

Consider the following SLS

$$
\Sigma:\left\{\begin{array}{l}
\dot{x}(t)=A\left(r_{t}\right) x(t)+B\left(r_{t}\right) u(t) \\
y(t)=C\left(r_{t}\right) x(t)+D\left(r_{t}\right) u(t)
\end{array}\right.
$$

where $x \in \mathbb{R}^{n}, u \in \mathbb{R}^{m}$ and $y \in \mathbb{R}^{p}$ are respectively the state, the input and the output (measurement) vectors and where $A(\cdot), B(\cdot), C(\cdot)$ and $D(\cdot)$ are real matrices of compatible dimensions. The exogenous and unobserved discrete mode variable (or switching signal) $r:[0, \infty) \rightarrow Q \stackrel{\text { def }}{=}$ $\{1, \ldots, N\}$, is assumed, as in (Babaali and Pappas, 2005), to be right-continuous and only a finite number of jumps can occur in any finite interval. It results that all the system trajectories are well defined. Without loss of generality and for the sake of homogeneity, all the inputs are assumed to be unknown. the control input signals, whose values are known, are considered to be measured i.e. we associate a virtual output equation of the form $y_{k^{\prime}}=u_{k}$ to these inputs. Moreover, our aim is to address, in the same framework autonomous SLS and SLS with known or unknown input. However, the discrete mode observability as it is defined in (De Santis et al., 2006), must be independent from the system's initial conditions $x_{0}$ and so cannot be satisfied for autonomous SLS. Indeed, for such systems case, if $x_{0}=0$, then $y(t) \equiv 0, \forall q \in Q$, and give no information about the discrete mode variable. To have a more general 
framework, we impose mode distinguishability for generic initial conditions $x_{0}$ and unknown input $u$ :

Definition 1 Two modes $q \in Q$ and $q^{\prime} \in Q$ with $\left(q \neq q^{\prime}\right)$ are distinguishable if one of following conditions holds:

- there exist an integer $s \geq 0$ and an expression $\Psi_{q}\left(y, \dot{y}, \ldots, y^{(s)}\right)=0$ satisfied for mode $q$ but not for mode $q^{\prime}$ for almost all initial conditions $x_{0}$ and input $u$,

- there exist an integer $s^{\prime} \geq 0$ and an expression $\Psi_{q^{\prime}}\left(y, \dot{y}, \ldots, y^{\left(s^{\prime}\right)}\right)=0$ satisfied for mode $q^{\prime}$ but not for mode $q$ for almost all initial conditions $x_{0}$ and input $u$.

Here, "for almost all initial conditions $x_{0}$ and input functions $u$ " is to be understood as "for all $\left(x_{0}^{T}, u^{T}\right)^{T} \in \mathbb{R}^{n+m}$ except for the zero set of some polynomial with real coefficients in the $n+m$ initial state and input components which constitutes a proper algebraic variety in the state and input space. Relatively to the definitions of (Babaali and Pappas, 2005), our notion of distinguishability of $q$ and $q^{\prime}$ is equivalent to the fact that $q$ is discernible from $q^{\prime}$ or viceversa. The mutual mode discernibility, which is a dissymmetric property in (Babaali and Pappas, 2005), is equivalent to have both conditions of Definition 1 satisfied.

Definition $2 S L S(\Sigma)$ is location observable if $\forall q \in Q$, $\forall q^{\prime} \in Q$, with $q \neq q^{\prime}$, $q$ and $q^{\prime}$ are distinguishable.

To establish the observability of SLS, we have to address, in addition to location observability, the state and input observability of each mode as defined in (Trentelman et al., 2001):

Definition 3 A continuous system is state and input observable if $y(t)=0, \forall t \geq 0$ implies $x(t)=0, \forall t \geq 0$ and $u(t)=0, \forall t>0$.

Roughly speaking, state and input observability is equivalent to the possibility to express the state and the unknown input in function of output and its time derivatives.

Definition 4 SLS $(\Sigma)$ is state and input observable if it is location observable and every mode is state and input observable.

Location observability analysis can be reduced to the study of the distinguishability of each pair of modes. Thus, there is no loss of generality in considering in the first part of the paper, for the sake of simplicity, that we have only two modes. At the end of Section 4, we extend the obtained results to the multiple modes case.

Since we study structural properties, it is pertinent to deal with structured systems, for which we assume that only the sparsity pattern of matrices $A(q), B(q), C(q)$ and $D(q)$ is known for $q \in\{1,2\}$. So, to each entry of these matrices, we only know whether its value is fixed to zero, or that it has an unknown real value represented by a real parameter $\lambda_{i}$. The vector of these parameters is $\Lambda=\left(\lambda_{1}, \lambda_{2}, \ldots, \lambda_{h}\right)^{T}$ and it is assumed that $\Lambda$ can take any value in $\mathbb{R}^{h}$. We denote by $A^{\lambda}(q), B^{\lambda}(q), C^{\lambda}(q)$ and $D^{\lambda}(q)$ respectively the matrices obtained by replacing the nonzeros in $A(q), B(q), C(q)$ and $D(q)$, for $q \in\{1,2\}$ by parameters $\lambda_{i}$ and we denote

$$
\Sigma_{\Lambda}:\left\{\begin{array}{l}
\dot{x}(t)=A^{\lambda}\left(r_{t}\right) x(t)+B^{\lambda}\left(r_{t}\right) u(t) \\
y(t)=C^{\lambda}\left(r_{t}\right) x(t)+D^{\lambda}\left(r_{t}\right) u(t)
\end{array}\right.
$$

If all parameters $\lambda_{i}$ are numerically fixed, we obtain a socalled admissible realization of $\operatorname{SSLS}\left(\Sigma_{\Lambda}\right)$. A property is true generically for SSLS $\left(\Sigma_{\Lambda}\right)$ if it is true for almost its realizations or equivalently for almost all parameters $\lambda_{i}$.
To study the location observability, it is pertinent and necessary to highlight the similarities and the differences between the models associated to these modes. Thus, we decompose each structured matrix into two parts: the first one is common to the two modes and the second one is specific to each mode i.e. for $q \in\{1,2\}, A^{\lambda}(q)=A_{0}^{\lambda}+A_{q}^{\lambda}, B^{\lambda}(q)=B_{0}^{\lambda}+B_{q}^{\lambda}$, $C^{\lambda}(q)=C_{0}^{\lambda}+C_{q}^{\lambda}$ and $D^{\lambda}(q)=D_{0}^{\lambda}+D_{q}^{\lambda}$. We assume that the entries of all these matrices are free.

\section{Graphical representation of structured switching lin- ear systems}

The digraph associated to $\left(\Sigma_{\Lambda}\right)$ is noted $\mathcal{G}\left(\Sigma_{\Lambda}\right)$. It is constituted by a vertex set $\mathcal{V}$ and an edge set $\mathcal{E}$. The vertices are associated to the internal continuous state, the input and the output components of $\left(\Sigma_{\Lambda}\right)$ and the directed edges represent links between these variables. More precisely, $\mathcal{V}=\mathbf{X} \cup \mathbf{U} \cup \mathbf{Y}$, where $\mathbf{X}=\left\{\mathbf{x}_{\mathbf{1}}, \ldots, \mathbf{x}_{\mathbf{n}}\right\}, \mathbf{U}=\left\{\mathbf{u}_{\mathbf{1}}, \ldots, \mathbf{u}_{\mathbf{m}}\right\}$ and $\mathbf{Y}=\left\{\mathbf{y}_{1}, \ldots, \mathbf{y}_{\mathbf{p}}\right\}$ are respectively the set of state, input and output vertices. For $q \in\{0,1,2\}$, we define $\mathcal{E}_{q}=A_{q}$-edges $\cup B_{q^{-}}$-dges $\cup C_{q^{-}}$-edges $\cup D_{q}$-edges, where, for $q \in\{0,1,2\}, A_{q}$-edges $=\left\{\left(\mathbf{x}_{\mathbf{j}}, \mathbf{x}_{\mathbf{i}}\right) \mid A_{q}(i, j) \neq 0\right\}$, $B_{q}$-edges $=\left\{\left(\mathbf{u}_{\mathbf{j}}, \mathbf{x}_{\mathbf{i}}\right) \mid B_{q}(i, j) \neq 0\right\}, C_{q}$-edges $=$ $\left\{\left(\mathbf{x}_{\mathbf{j}}, \mathbf{y}_{\mathbf{i}}\right) \mid C_{q}(i, j) \neq 0\right\} \quad$ and $D_{q}$-edges $=$ $\left\{\left(\mathbf{u}_{\mathbf{j}}, \mathbf{y}_{\mathbf{i}}\right) \mid D_{q}(i, j) \neq 0\right\}$. Finally the edge set is $\mathcal{E}=\bigcup_{q=0}^{2} \mathcal{E}_{q}$. The edges included in $\mathcal{E}_{0}$ represent the common part of the two modes' models, while $\mathcal{E}_{q}$, for $q \in\{1,2\}$ is related to the specific part of each mode's model. Each edge is associated to a free non-zero parameter of the system's model called the weight of the edge. A number $q$ is written under each $\mathcal{E}_{q}$-edge.

Example 1 To the system defined by the following matrices, we associate the digraph in Figure 1.

$\begin{aligned} A_{0}^{\lambda}= & \left(\begin{array}{cccccccc}0 & 0 & 0 & 0 & 0 & 0 & 0 & 0 \\ 0 & 0 & \lambda_{1} & 0 & 0 & 0 & 0 & 0 \\ 0 & \lambda_{2} & 0 & 0 & 0 & 0 & 0 & 0 \\ 0 & 0 & 0 & 0 & \lambda_{3} & 0 & 0 & \lambda_{4} \\ 0 & \lambda_{5} & 0 & 0 & 0 & 0 & 0 & 0 \\ 0 & 0 & 0 & 0 & 0 & 0 & 0 & 0 \\ 0 & 0 & 0 & 0 & \lambda_{6} & \lambda_{7} & 0 & 0 \\ 0 & 0 & 0 & 0 & 0 & 0 & \lambda_{8} & 0\end{array}\right), B_{0}^{\lambda}=\left(\begin{array}{c}0 \\ 0 \\ 0 \\ 0 \\ 0 \\ \lambda_{12} \\ 0 \\ 0\end{array}\right), B_{1}^{\lambda}=B_{2}^{\lambda}= \\ 0, C_{0}^{\lambda}= & \left(\begin{array}{cccccccc}\lambda_{13} & 0 & 0 & 0 & 0 & 0 & 0 & 0 \\ 0 & 0 & 0 & 0 & \lambda_{14} & 0 & 0 & 0 \\ 0 & 0 & 0 & \lambda_{15} & 0 & 0 & 0 & 0 \\ 0 & 0 & 0 & 0 & 0 & 0 & 0 & \lambda_{16}\end{array}\right), \text { all the entries of } A_{1}^{\lambda}\end{aligned}$ are zero except $A_{1}^{\lambda}(1,2)=\lambda_{9}$, all the entries of $A_{2}^{\lambda}$ are zero except $A_{2}^{\lambda}(5,2)=\lambda_{10}$ and $A_{2}^{\lambda}(7,6)=\lambda_{11}$, the elements of matrices $C_{1}^{\lambda}, C_{2}^{\lambda}, D_{0}^{\lambda}, D_{1}^{\lambda}$ and $D_{2}^{\lambda}$ are equal to zero.

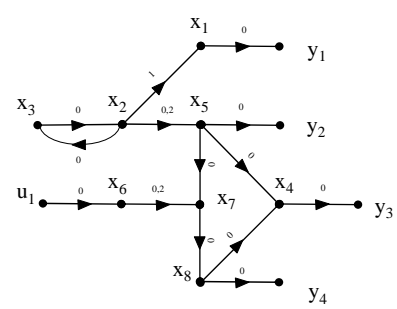

Figure 1. Digraph associated to system of Example 1 
The digraph representing the SSLS is built from the superposition of the digraphs related to each mode. To study the properties of the system associated to a specific mode $q$, we have to restrict the edge set to $\mathcal{E}_{0} \cup \mathcal{E}_{q}$. Then, many of the functions and specific vertex subsets, defined below, present an index $q$ related to the considered mode.

- Two edges $e_{1}=\left(\mathbf{v}_{\mathbf{1}}, \mathbf{v}_{\mathbf{1}}^{\prime}\right)$ and $e_{2}=\left(\mathbf{v}_{\mathbf{2}}, \mathbf{v}_{\mathbf{2}}^{\prime}\right)$ are $v$-disjoint if $\mathbf{v}_{\mathbf{1}} \neq \mathbf{v}_{\mathbf{2}}$ and $\mathbf{v}_{\mathbf{1}}^{\prime} \neq \mathbf{v}_{\mathbf{2}}^{\prime}$. Some edges are $v$-disjoint if they are mutually $v$-disjoint.

- A path $P$ is denoted $P=\mathbf{v}_{\mathbf{s}_{0}} \rightarrow \mathbf{v}_{\mathbf{s}_{1}} \rightarrow \ldots \rightarrow \mathbf{v}_{\mathbf{s}_{i}}$, where $\left(\mathbf{v}_{\mathbf{s}_{\mathbf{j}}}, \mathbf{v}_{\mathbf{s}_{\mathbf{j}+\mathbf{1}}}\right) \in \mathcal{E}$ for $j=0,1, \ldots, i-1$. We say in this case that $P$ covers $\mathbf{v}_{\mathbf{s}_{0}}, \mathbf{v}_{\mathbf{s}_{1}}, \ldots, \mathbf{v}_{\mathbf{s}_{\mathbf{i}}}$. A path is simple when every vertex occurs only once in this path. The weight of $P$ is the product of the weights of all its edges.

- A cycle is a path of the form $\mathbf{v}_{\mathbf{s}_{0}} \rightarrow \mathbf{v}_{\mathbf{s}_{1}} \rightarrow \ldots \rightarrow \mathbf{v}_{\mathbf{s}_{\mathbf{i}}} \rightarrow$ $\mathbf{v}_{\mathbf{s}_{0}}$, where $\mathbf{v}_{\mathbf{s}_{0}}, \mathbf{v}_{\mathbf{s}_{1}}, \ldots, \mathbf{v}_{\mathbf{s}_{\mathbf{i}}}$ are distinct.

- For $q \in\{1,2\}$, we say that path $P$ is included in $\mathcal{E}_{0} \cup \mathcal{E}_{q}$ if all its edges are included in $\mathcal{E}_{0} \cup \mathcal{E}_{q}$.

- Some paths (resp. cycles) are disjoint if they have no common vertex. A set of disjoint cycles is called a cycle family. - $P$ is a $\mathbf{Y}$-topped path if its end belongs to $\mathbf{Y}$. A $\mathbf{Y}$-topped path family is a set of disjoint simple $\mathbf{Y}$-topped paths.

- The union of a $\mathbf{Y}$-topped path family and a cycle family is disjoint if they have no common vertex. Such union covers vertex $\mathbf{v}$ if it contains a path or a cycle which covers $\mathbf{v}$. In the sequel, $\mathcal{V}_{1}$ and $\mathcal{V}_{2}$ represent two subsets of $\mathcal{V}$. We denote by $\operatorname{card}(\cdot)$ the cardinality function and $\mathcal{V}_{1} \backslash \mathcal{V}_{2}$ is the set of elements in $\mathcal{V}_{1}$ which are not in $\mathcal{V}_{2}$.

- A path $P=\mathbf{v}_{\mathbf{s}_{0}} \rightarrow \mathbf{v}_{\mathbf{s}_{1}} \rightarrow \ldots \rightarrow \mathbf{v}_{\mathbf{s}_{\mathbf{i}}}$ is said a $\mathcal{V}_{1}$ $\mathcal{V}_{2}$ path if $\mathbf{v}_{\mathbf{s}_{\mathbf{o}}} \in \mathcal{V}_{1}$ and $\mathbf{v}_{\mathbf{s}_{\mathbf{i}}} \in \mathcal{V}_{2}$. If the only vertex of $P$ which belongs to $\mathcal{V}_{1}$ is $\mathbf{v}_{\mathbf{s}_{0}}$ and the only vertex of $P$ which belongs to $\mathcal{V}_{2}$ is $\mathbf{v}_{\mathbf{s}_{\mathbf{i}}}, P$ is called a direct $\mathcal{V}_{1}-\mathcal{V}_{2}$ path.

For $q=\{1,2\}$, we denote:

- $\rho_{q}\left[\mathcal{V}_{1}, \mathcal{V}_{2}\right]$ is the maximal number of disjoint $\mathcal{V}_{1}-\mathcal{V}_{2}$ paths included in $\mathcal{E}_{0} \cup \mathcal{E}_{q}$. A set of $\rho_{q}\left[\mathcal{V}_{1}, \mathcal{V}_{2}\right]$ disjoint $\mathcal{V}_{1}-\mathcal{V}_{2}$ paths included in $\mathcal{E}_{0} \cup \mathcal{E}_{q}$ is a maximum $\mathcal{V}_{1}-\mathcal{V}_{2}$ linking in $\mathcal{E}_{0} \cup \mathcal{E}_{q}$. - $\mu_{q}\left[\mathcal{V}_{1}, \mathcal{V}_{2}\right]$ is the minimal number of vertices of $\mathbf{U} \cup \mathbf{X} \cup \mathbf{Y}$ belonging to a maximum $\mathcal{V}_{1}-\mathcal{V}_{2}$ linking included in $\mathcal{E}_{0} \cup \mathcal{E}_{q}$. - $V_{\text {ess }, q}\left[\mathcal{V}_{1}, \mathcal{V}_{2}\right]$ is the subset including the vertices present in all the maximum $\mathcal{V}_{1}-\mathcal{V}_{2}$ linkings included in $\mathcal{E}_{0} \cup \mathcal{E}_{q}$.

- $\theta_{q}\left[\mathcal{V}_{1}, \mathcal{V}_{2}\right]$ is the maximal number of $v$-disjoint edges included in $\mathcal{E}_{0} \cup \mathcal{E}_{q}$, starting in $\mathcal{V}_{1}$ and ending in $\mathcal{V}_{2}$.

- There exists a unique vertex subset $S_{q}^{o}\left[\mathcal{V}_{1}, \mathcal{V}_{2}\right]$ and called minimum output separator which is the set of begin vertices of all direct $V_{\text {ess }, q}\left[\mathcal{V}_{1}, \mathcal{V}_{2}\right]-\mathcal{V}_{2}$ paths included in $\mathcal{E}_{0} \cup \mathcal{E}_{q}$.

In Example $1, \rho_{1}[\mathbf{U}, \mathbf{Y}]=\rho_{2}[\mathbf{U}, \mathbf{Y}]=1, \mu_{1}[\mathbf{U}, \mathbf{Y}]=$ $\mu_{2}[\mathbf{U}, \mathbf{Y}]=5 . V_{e s s, 1}[\mathbf{U}, \mathbf{Y}]=V_{e s s, 2}[\mathbf{U}, \mathbf{Y}]=$ $\left\{\mathbf{u}_{\mathbf{1}}, \mathbf{x}_{\mathbf{6}}, \mathbf{x}_{\mathbf{7}}, \mathbf{x}_{\mathbf{8}}\right\}$. Finally, $S_{1}^{o}[\mathbf{U}, \mathbf{Y}]=\left\{\mathbf{x}_{\mathbf{8}}\right\}$.

Functions $\theta_{q}, \rho_{q}$ and $\mu_{q}$ and the two vertex subsets $V_{e s s, q}$ and $S_{q}^{o}$ constitute the classical graphic-notions used in most of the results related to the structural analysis using graphtheoretic approach. Indeed, for example, $\theta_{q}(\mathbf{X}, \mathbf{Y})$ is equal to the generic rank of matrix $C_{q}$ (Murota, 1987; Reinschke, 1988). $\rho_{q}$ and $\mu_{q}$ are associated to the generic rank of transfer matrices.

\section{Main results}

\subsection{Preliminaries}

Let $\mathbf{V}_{\mathbf{Y}} \subseteq \mathbf{Y}$ denotes a vertex subset which plays the role of the output vertex subset instead of Y. Our aim is to char- acterize the generic dimension of the observability subspace when only measurements related to $\mathbf{V}_{\mathbf{Y}}$ are available.

Definition 5 Consider SSLS $\left(\Sigma_{\Lambda}\right)$ associated to digraph $\mathcal{G}\left(\Sigma_{\Lambda}\right)$. For each vertex subset $\mathbf{V}_{\mathbf{Y}} \subseteq \cup \mathbf{Y}$ and for each $q \in\{1,2\}$, we define the following subdivision:

- $\mathbf{X}_{\mathbf{1}, \mathbf{q}}\left(\mathbf{V}_{\mathbf{Y}}\right) \stackrel{\text { def }}{=}\left\{\mathbf{x}_{\mathbf{i}} \mid \rho_{q}\left[\mathbf{U} \cup\left\{\mathbf{x}_{\mathbf{i}}\right\}, \mathbf{V}_{\mathbf{Y}}\right]>\rho_{q}\left[\mathbf{U}, \mathbf{V}_{\mathbf{Y}}\right]\right\}$;

- $\mathbf{Y}_{\mathbf{0}, \mathbf{q}}\left(\mathbf{V}_{\mathbf{Y}}\right) \stackrel{\text { def }}{=} \mathbf{V}_{\mathbf{Y}} \cap V_{\text {ess }, q}\left[\mathbf{U}, \mathbf{V}_{\mathbf{Y}}\right]$;

- $\mathbf{Y}_{\mathbf{1}, \mathbf{q}}\left(\mathbf{V}_{\mathbf{Y}}\right) \stackrel{\text { def }}{=} \mathbf{V}_{\mathbf{Y}} \backslash \mathbf{Y}_{\mathbf{0}, \mathbf{q}}\left(\mathbf{V}_{\mathbf{Y}}\right)$;

- $\mathbf{U}_{\mathbf{0}, \mathbf{q}}\left(\mathbf{V}_{\mathbf{Y}}\right) \stackrel{\text { def }}{=}\left\{\mathbf{u}_{\mathbf{i}} \mid \theta_{q}\left[\left\{\mathbf{u}_{\mathbf{i}}\right\}, \mathbf{X}_{\mathbf{1}, \mathbf{q}}\left(\mathbf{V}_{\mathbf{Y}}\right) \cup \mathbf{Y}_{\mathbf{1}, \mathbf{q}}\left(\mathbf{V}_{\mathbf{Y}}\right)\right]=0\right\}$;

- $\mathbf{U}_{\mathbf{1}, \mathbf{q}}\left(\mathbf{V}_{\mathbf{Y}}\right) \stackrel{\text { def }}{=} \mathbf{U} \backslash \mathbf{U}_{\mathbf{0}, \mathbf{q}}\left(\mathbf{V}_{\mathbf{Y}}\right)$;

- $\mathbf{X}_{\mathbf{s}, \mathbf{q}}\left(\mathbf{V}_{\mathbf{Y}}\right) \stackrel{\text { def }}{=} S_{q}^{o}\left[\mathbf{U}_{\mathbf{0}, \mathbf{q}}\left(\mathbf{V}_{\mathbf{Y}}\right), \mathbf{V}_{\mathbf{Y}}\right] \cap \mathbf{X}$;

- $\mathbf{X}_{\mathbf{0}, \mathbf{q}}\left(\mathbf{V}_{\mathbf{Y}}\right) \stackrel{\text { def }}{=} \mathbf{X} \backslash\left(\mathbf{X}_{\mathbf{1}, \mathbf{q}}\left(\mathbf{V}_{\mathbf{Y}}\right) \cup \mathbf{X}_{\mathbf{s}, \mathbf{q}}\left(\mathbf{V}_{\mathbf{Y}}\right)\right)$.

One of the main properties of the subdivision (which are proved in Lemma 5 of (Boukhobza et al., 2007)) is that there is no edge from $\mathbf{X}_{\mathbf{0 , q}}\left(\mathbf{V}_{\mathbf{Y}}\right) \cup \mathbf{U}_{\mathbf{0 , q}}\left(\mathbf{V}_{\mathbf{Y}}\right)$ to $\mathbf{X}_{\mathbf{1}, \mathbf{q}}\left(\mathbf{V}_{\mathbf{Y}}\right) \cup \mathbf{Y}_{\mathbf{1}, \mathbf{q}}\left(\mathbf{V}_{\mathbf{Y}}\right)$ and so that the dynamics of the components included in $\mathbf{X}_{\mathbf{1}, \mathbf{q}}\left(\mathbf{V}_{\mathbf{Y}}\right)$ is independent from the elements $\mathbf{X}_{\mathbf{0}, \mathbf{q}}\left(\mathbf{V}_{\mathbf{Y}}\right) \cup \mathbf{U}_{\mathbf{0}, \mathbf{q}}\left(\mathbf{V}_{\mathbf{Y}}\right)$. We can then decompose for each mode the system into two subsystems: $\left(\Sigma_{0, q}\right)$ defined by input $\mathbf{U}_{\mathbf{0}, \mathbf{q}}\left(\mathbf{V}_{\mathbf{Y}}\right)$, state $\mathbf{X}_{\mathbf{0}, \mathbf{q}}\left(\mathbf{V}_{\mathbf{Y}}\right)$ and output $\mathbf{X}_{\mathbf{s}, \mathbf{q}}\left(\mathbf{V}_{\mathbf{Y}}\right) \cup \mathbf{Y}_{\mathbf{0}, \mathbf{q}}\left(\mathbf{V}_{\mathbf{Y}}\right)$ and $\left(\Sigma_{1, q}\left(V_{Y}\right)\right)$, having more outputs than inputs, defined by input $\mathbf{U}_{\mathbf{1}, \mathbf{q}}\left(\mathbf{V}_{\mathbf{Y}}\right)$ and $\mathbf{X}_{\mathbf{s}, \mathbf{q}}\left(\mathbf{V}_{\mathbf{Y}}\right)$, state $\mathbf{X}_{\mathbf{1}, \mathbf{q}}\left(\mathbf{V}_{\mathbf{Y}}\right)$ and output $\mathbf{Y}_{\mathbf{1}, \mathbf{q}}\left(\mathbf{V}_{\mathbf{Y}}\right)$.

In Example 1, $\mathbf{X}_{1,1}(\mathbf{Y})=\left\{\mathbf{x}_{1}, \mathbf{x}_{\mathbf{2}}, \mathbf{x}_{\mathbf{3}}, \mathbf{x}_{\mathbf{4}}, \mathbf{x}_{\mathbf{5}}\right\}$, $\mathbf{Y}_{\mathbf{1}, \mathbf{1}}(\mathbf{Y})=\mathbf{Y}, \mathbf{Y}_{\mathbf{0 , 1}}(\mathbf{Y})=\emptyset=\mathbf{U}_{\mathbf{1 , 1}, \mathbf{1}}(\mathbf{Y}), \mathbf{U}_{\mathbf{0 , 1}}(\mathbf{Y})=$ $\left\{\mathbf{u}_{\mathbf{1}}\right\}, \mathbf{X}_{\mathbf{s}, \mathbf{1}}(\mathbf{Y})=\left\{\mathbf{x}_{\mathbf{8}}\right\}$ and $\mathbf{X}_{\mathbf{0}, \mathbf{1}}(\mathbf{Y})=\left\{\mathbf{x}_{\mathbf{6}}, \mathbf{x}_{\mathbf{7}}\right\}$.

For $\mathbf{V}_{\mathbf{Y}}=\mathbf{Y}$ and for each $q \in\{1,2\}$ we can write system $\left(\Sigma_{\Lambda}\right)$ in mode $q$ as (Boukhobza et al., 2007):

$$
\left\{\begin{aligned}
\dot{X}_{0, q}= & A_{0,0}(q) X_{0, q}+A_{0, s}(q) X_{s, q}+A_{0,1}(q) X_{1, q}+ \\
& +B_{0,0}(q) U_{0, q}+B_{0,1}(q) U_{1, q} \\
\dot{X}_{s, q}= & A_{s, 0}(q) X_{0, q}+A_{s, s} X_{s, q}+A_{s, 1}(q) X_{1, q}+ \\
& B_{s, 0}(q) U_{0, q}++B_{s, 1}(q) U_{1, q} \\
\dot{X}_{1, q}= & A_{1, s}(q) X_{s, q}+A_{1,1}(q) X_{1, q}+B_{1,1}(q) U_{1, q} \\
Y_{0, q}= & C_{0,0}(q) X_{0, q}+C_{0, s}(q) X_{s, q}+C_{0,1}(q) X_{1, q}+ \\
& +D_{0,0}(q) U_{0, q}+D_{0,1}(q) U_{1, q} \\
Y_{1, q}= & C_{1, s}(q) X_{s, q}+C_{1,1}(q) X_{1, q}+D_{1,1}(q) U_{1, q}
\end{aligned}\right.
$$

where $X_{0, q}, X_{s, q}, X_{1, q}, U_{0, q}, U_{1, q}, Y_{0, q}$ and $Y_{1, q}$ represent the state, the unknown input and the output associated to vertex subsets $\mathbf{X}_{\mathbf{0}, \mathbf{q}}(\mathbf{Y}), \mathbf{X}_{\mathbf{s}, \mathbf{q}}(\mathbf{Y}), \mathbf{X}_{\mathbf{1}, \mathbf{q}}(\mathbf{Y}), \mathbf{U}_{\mathbf{0}, \mathbf{q}}(\mathbf{Y})$, $\mathbf{U}_{\mathbf{1}, \mathbf{q}}(\mathbf{Y}), \mathbf{Y}_{\mathbf{0}, \mathbf{q}}(\mathbf{Y})$ and $\mathbf{Y}_{\mathbf{1}, \mathbf{q}}(\mathbf{Y})$ respectively.

In (van der Woude et al., 2003; van der Woude, 2000), the authors consider the following structured linear system: $\left\{\begin{array}{l}\dot{x}(t)=A^{\lambda} x(t)+B^{\lambda} u(t) \\ y(t)=C^{\lambda} x(t)+D^{\lambda} u(t)\end{array}\right.$ and they study its pencil matrix defined by: $P(s)=\left(\begin{array}{cc}A^{\lambda}-s I_{n} & B^{\lambda} \\ C^{\lambda} & D^{\lambda}\end{array}\right)$. Regarding $P(s)$ as a rational matrix, its generic rank, denoted $g \_r a n k(P(s))$, is such that $g \_r a n k(P(s))=r, \forall s \in \mathbb{C}$ means that for almost all parameter values, $\operatorname{rank}(P(s))=r, \forall s \in \mathbb{C}$. Important remarks are summarized in the following lemmas:

Lemma 1 There cannot exist an equation linking only the output components of square subsystem $\Sigma_{0, q}, Y_{0, q}$ and $X_{s, q}$. Proof: Using results shown in (van der Woude, 2000) (Theorem 5.1), we have that the generic number of invariant zeros of the pencil matrix of system $\Sigma_{0}$ is equal to $\operatorname{card}\left(\mathbf{X}_{\mathbf{0}, \mathbf{q}}\right)+\operatorname{card}\left(\mathbf{X}_{\mathbf{s}, \mathbf{q}}\right)+$ 
$\operatorname{card}\left(\mathbf{U}_{\mathbf{0}, \mathbf{q}}\right)-\left(\mu_{q}\left[\mathbf{U}_{\mathbf{0}, \mathbf{q}}(\mathbf{Y}), \mathbf{X}_{\mathbf{s}, \mathbf{q}}(\mathbf{Y}) \cup \quad \mathbf{Y}_{\mathbf{0}, \mathbf{q}}(\mathbf{Y})\right]-\right.$ $\left.\rho_{q}\left[\mathbf{U}_{\mathbf{0}, \mathbf{q}}(\mathbf{Y}), \mathbf{X}_{\mathbf{s}, \mathbf{q}}(\mathbf{Y}) \cup \mathbf{Y}_{\mathbf{0}, \mathbf{q}}(\mathbf{Y})\right]+\operatorname{card}\left(\mathbf{X}_{\mathbf{s}, \mathbf{q}}\right)\right)$. Thus, the dimension of the strongly observable subspace and so the number of possible independent observation equations is equal to $\mu_{q}\left[\mathbf{U}_{\mathbf{0}, \mathbf{q}}(\mathbf{Y}), \mathbf{X}_{\mathbf{s}, \mathbf{q}}(\mathbf{Y}) \cup \mathbf{Y}_{\mathbf{0}, \mathbf{q}}(\mathbf{Y})\right]-$ $\rho_{q}\left[\mathbf{U}_{\mathbf{0}, \mathbf{q}}(\mathbf{Y}), \mathbf{X}_{\mathbf{s}, \mathbf{q}}(\mathbf{Y}) \cup \mathbf{Y}_{\mathbf{0}, \mathbf{q}}(\mathbf{Y})\right]$. But, the cardinality of $\mathbf{U}_{\mathbf{0}, \mathbf{q}}(\mathbf{Y}) \cup \mathbf{X}_{\mathbf{0}, \mathbf{q}}(\mathbf{Y})$, which represents the unknown variables for this system, is at least $\mu_{q}\left[\mathbf{U}_{\mathbf{0 , q}}(\mathbf{Y}), \mathbf{X}_{\mathbf{s}, \mathbf{q}}(\mathbf{Y}) \cup\right.$ $\left.\mathbf{Y}_{\mathbf{0}, \mathbf{q}}(\mathbf{Y})\right]-\rho_{q}\left[\mathbf{U}_{\mathbf{0}, \mathbf{q}}(\mathbf{Y}), \mathbf{X}_{\mathbf{s}, \mathbf{q}}(\mathbf{Y}) \cup \mathbf{Y}_{\mathbf{0}, \mathbf{q}}(\mathbf{Y})\right] . \triangle$

Lemma 2 For subsystem $\left(\Sigma_{1, q}\right)$, for each $\mathbf{Y}_{\mathbf{u}}=$ $\left\{\mathbf{y}_{\mathbf{i}_{1, \mathbf{q}}}, \mathbf{y}_{\mathbf{i}_{\mathbf{2}}}, \ldots, \mathbf{y}_{\mathbf{i}_{\mathbf{k}}}\right\} \subset \mathbf{Y}_{\mathbf{1}, \mathbf{q}}(\mathbf{Y})$ such that $\rho_{q}\left[\mathbf{U}_{\mathbf{1}, \mathbf{q}}(\mathbf{Y}) \cup\right.$ $\left.\mathbf{X}_{\mathbf{s}, \mathbf{q}}(\mathbf{Y}), \mathbf{Y}_{\mathbf{u}}\right]=\operatorname{card}\left(\mathbf{U}_{\mathbf{1}, \mathbf{q}}(\mathbf{Y}) \cup \mathbf{X}_{\mathbf{s}, \mathbf{q}}(\mathbf{Y})\right)$, there exist generically a matrix $G$, a function $\varphi$ and an integer $\nu \leq n_{1, q}$ such that $\left(X_{s, q}^{T}, U_{1, q}^{T}\right)^{T}=\varphi\left(Y_{u}, \dot{Y}_{u}, \ldots, Y_{u}^{(\nu)}\right)+G X_{1, q}$, where $Y_{u}^{(\nu)}=\left(y_{i_{1, q}}^{(\nu)}, y_{i_{2}}^{(\nu)}, \ldots, y_{i_{k}}^{(\nu)}\right)^{T}$

Proof: According to (Dion et al., 2003) (Theorem 4), for $\left(\Sigma_{1, q}\right)$, the existence of such $\mathbf{Y}_{\mathbf{u}}$ implies the invertibility of $\left(\Sigma_{1, q}\right)$ using only $Y_{u}$. Thus, using the inversion algorithm of (Silverman, 1969), we can express the input of such system i.e. $U_{1, q}$ and $X_{s, q}$ in function of its state $X_{1, q}$ and its output components $Y_{u}$ and their derivatives.

$\triangle$

Definition 6 Consider SSLS $\left(\Sigma_{\Lambda}\right)$ associated to digraph $\mathcal{G}\left(\Sigma_{\Lambda}\right)$. To each vertex subset $\mathbf{V}_{\mathbf{Y}} \subseteq \mathbf{Y}$ and $q \in\{1,2\}$, we associate the integer $\beta_{q}\left(\mathbf{V}_{\mathbf{Y}}\right)$ defined as $\mu_{q}\left[\mathbf{U}_{\mathbf{0}, \mathbf{q}}\left(\mathbf{V}_{\mathbf{Y}}\right), \mathbf{X}_{\mathbf{s}, \mathbf{q}}\left(\mathbf{V}_{\mathbf{Y}}\right) \cup \mathbf{Y}_{\mathbf{0}, \mathbf{q}}\left(\mathbf{V}_{\mathbf{Y}}\right)\right]-$ $\rho_{q}\left[\mathbf{U}_{\mathbf{0}, \mathbf{q}}\left(\mathbf{V}_{\mathbf{Y}}\right), \mathbf{X}_{\mathbf{s}, \mathbf{q}}\left(\mathbf{V}_{\mathbf{Y}}\right) \cup \mathbf{Y}_{\mathbf{0}, \mathbf{q}}\left(\mathbf{V}_{\mathbf{Y}}\right)\right]$ plus the maximal number of vertices of $\mathbf{X}_{\mathbf{1}, \mathbf{q}}\left(\mathbf{V}_{\mathbf{Y}}\right) \cup \mathbf{U}_{\mathbf{1 , q}}\left(\mathbf{V}_{\mathbf{Y}}\right) \cup \mathbf{X}_{\mathbf{s}, \mathbf{q}}\left(\mathbf{V}_{\mathbf{Y}}\right)$ covered by a disjoint union of

- $a \mathbf{U}_{\mathbf{1}, \mathbf{q}}\left(\mathbf{V}_{\mathbf{Y}}\right) \cup \mathbf{X}_{\mathbf{s}, \mathbf{q}}\left(\mathbf{V}_{\mathbf{Y}}\right)-\mathbf{Y}_{\mathbf{1}, \mathbf{q}}\left(\mathbf{V}_{\mathbf{Y}}\right)$ linking of maximal size;

- a $\mathbf{Y}_{\mathbf{1}, \mathbf{q}}\left(\mathbf{V}_{\mathbf{Y}}\right)$-topped path family ;

- a cycle family covering only elements of $\mathbf{X}_{\mathbf{1}, \mathbf{q}}\left(\mathbf{V}_{\mathbf{Y}}\right)$.

Lemma 3 Consider SSLS $\left(\Sigma_{\Lambda}\right)$ represented by digraph $\mathcal{G}\left(\Sigma_{\Lambda, \text { lin }}\right), \beta_{q}\left(\mathbf{V}_{\mathbf{Y}}\right)$ is equal to the generic dimension of the observable subspace in the extended state and input space $\left(x^{T}(t), u^{T}(t)\right)^{T}$ for mode $q$ and restricting the measurements to the output components associated to $\mathbf{V}_{\mathbf{Y}}$.

Proof: Using results proved in (van der Woude, 2000) ( Theorems 5.1 and 5.2) and in (van der Woude et al., 2003) (Theorem 3.6) and considering that the measurements of the system are reduced to the components associated to the elements of $\mathbf{V}_{\mathbf{Y}}$, for each mode $q$, number $\beta_{q}\left(\mathbf{V}_{\mathbf{Y}}\right)$ is equal to $n+m-g_{-} n_{q, i n v, z}\left(P_{q}(s)\right)$, where $g \_n_{q, i n v, z}\left(P_{q}(s)\right)$ denotes the generic number of invariant zeros of the pencil matrix $P_{q}(s)$ associated to mode $q$. Moreover, from (Trentelman et al., 2001) (Lemma 7.7), we have that $n+m-g \_n_{q, i n v, z}\left(P_{q}(s)\right)$ is also equal to the dimension of the observable subspace, i.e. the number of observable directions or independent state and input combinations, in the extended state and input space.

\subsection{Input and state observability of each mode}

To establish the state and input observability of each mode, we use immediately Corollary 7 of (Boukhobza et al., 2007):

Proposition 1 Each mode of SSLS $\left(\Sigma_{\Lambda}\right)$ is generically state and input observable iff in digraph $\mathcal{G}\left(\Sigma_{\Lambda}\right)$

i. $\theta_{q}(\mathbf{X} \cup \mathbf{U}, \mathbf{X} \cup \mathbf{Y})=n+m$; ii. $\mathbf{X}_{\mathbf{0 , q}}(\mathbf{Y}) \cup \mathbf{U}_{\mathbf{0 , q}}(\mathbf{Y}) \subseteq V_{e s s, q}\left[\mathbf{U}_{\mathbf{0 , q}}(\mathbf{Y}), \mathbf{Y}_{\mathbf{0 , q}}(\mathbf{Y}) \cup\right.$ $\left.\mathbf{X}_{\mathbf{s}, \mathbf{q}}(\mathbf{Y})\right]$.

In Example 1, for mode 1, all edges $\left(\mathbf{u}_{\mathbf{1}}, \mathbf{x}_{\mathbf{6}}\right),\left(\mathbf{x}_{\mathbf{1}}, \mathbf{y}_{\mathbf{1}}\right)$, $\left(\mathrm{x}_{2}, \mathrm{x}_{3}\right),\left(\mathrm{x}_{3}, \mathrm{x}_{2}\right),\left(\mathrm{x}_{4}, \mathrm{y}_{3}\right),\left(\mathrm{x}_{5}, \mathrm{y}_{2}\right),\left(\mathrm{x}_{6}, \mathrm{x}_{7}\right),\left(\mathrm{x}_{7}, \mathrm{x}_{8}\right)$ and $\left(\mathbf{x}_{\mathbf{8}}, \mathbf{y}_{\mathbf{4}}\right)$ are $v$-disjoint. Moreover, $\mathbf{X}_{\mathbf{0}, \mathbf{1}}(\mathbf{Y}) \cup$ $\mathbf{U}_{\mathbf{0 , 1}}(\mathbf{Y})=\left\{\mathbf{u}_{\mathbf{1}}, \mathbf{x}_{\mathbf{6}}, \mathbf{x}_{\mathbf{7}}\right\} \subseteq V_{\text {ess }, 1}\left[\left[\mathbf{U}_{\mathbf{0}, \mathbf{q}}(\mathbf{Y}), \mathbf{Y}_{\mathbf{0}, \mathbf{q}}(\mathbf{Y}) \cup\right.\right.$ $\left.\left.\mathbf{X}_{\mathbf{s}, \mathbf{q}}(\mathbf{Y})\right]\right]=\left\{\mathbf{u}_{\mathbf{1}}, \mathbf{x}_{\mathbf{6}}, \mathbf{x}_{\mathbf{7}}, \mathbf{x}_{\mathbf{8}}\right\}$. Thus, mode 1 is generically input and state observable.

\subsection{Location observability analysis}

Using the previous settings and definitions, we analyse, hereafter, the existence of an algebraic equation depending on the mode to achieve mode distinguishability.

Proposition $2 \operatorname{SSLS}\left(\Sigma_{\Lambda}\right)$, with two possible modes $q \in$ $\{1,2\}$, associated to digraph $\mathcal{G}\left(\Sigma_{\Lambda}\right)$ is generically location observable iff one of the following conditions is satisfied:

Cond1. For some $q \in\{1,2\}$, there exists a cycle $\mathcal{C}$ included in $\mathcal{E}_{0} \cup \mathcal{E}_{q}$, covering only elements of $\mathbf{X}_{\mathbf{1}, \mathbf{q}}(\mathbf{Y})$ and containing at least one edge of $\mathcal{E}_{q}$;

Cond2. For some $q \in\{1,2\}$, there exists a direct $\mathbf{X}_{\mathbf{s}, \mathbf{q}}(\mathbf{Y}) \cup \mathbf{U}_{\mathbf{1}, \mathbf{q}}(\mathbf{Y})-\mathbf{Y}_{\mathbf{1}, \mathbf{q}}(\mathbf{Y})$ path included in $\mathcal{E}_{0} \cup \mathcal{E}_{q}$ and containing at least one edge of $\mathcal{E}_{q}$;

Cond3. For some $q \in\{1,2\}$, there exist a vertex $\mathbf{y}_{\mathbf{i}} \in \mathbf{Y}_{\mathbf{1}, \mathbf{q}}(\mathbf{Y})$ and disjoint vertex subsets $\tilde{\mathbf{Y}} \subseteq \mathbf{Y}_{\mathbf{1}, \mathbf{q}}(\mathbf{Y}) \backslash\left\{\mathbf{y}_{\mathbf{i}}\right\}$ (which can be empty), $\mathbf{Y}_{\mathbf{u}} \subseteq \mathbf{Y}_{\mathbf{1}, \mathbf{q}}(\mathbf{Y}) \backslash\left\{\mathbf{y}_{\mathbf{i}}\right\}$ such that:

$\diamond \rho_{q}\left[\mathbf{U}_{\mathbf{1}, \mathbf{q}}(\mathbf{Y}) \cup \mathbf{X}_{\mathbf{s}, \mathbf{q}}(\mathbf{Y}), \mathbf{Y}_{\mathbf{u}}\right]=\operatorname{card}\left(\mathbf{U}_{\mathbf{1}, \mathbf{q}}(\mathbf{Y}) \cup \mathbf{X}_{\mathbf{s}, \mathbf{q}}(\mathbf{Y})\right)$,

$\diamond$ For some $q^{\prime} \in\{1,2\}, q^{\prime} \neq q, \beta_{q}\left(\tilde{\mathbf{Y}} \cup\left\{\mathbf{y}_{\mathbf{i}}\right\} \cup \mathbf{Y}_{\mathbf{u}}\right)-\beta_{q}(\tilde{\mathbf{Y}} \cup$ $\left.\mathbf{Y}_{\mathbf{u}}\right) \neq \beta_{q^{\prime}}\left(\tilde{\mathbf{Y}} \cup\left\{\mathbf{y}_{\mathbf{i}}\right\} \cup \mathbf{Y}_{\mathbf{u}}\right)-\beta_{q^{\prime}}\left(\tilde{\mathbf{Y}} \cup \mathbf{Y}_{\mathbf{u}}\right)$ or $\mathbf{y}_{\mathbf{i}} \notin \mathbf{Y}_{\mathbf{1}, \mathbf{q}^{\prime}}(\mathbf{Y})$. Cond4. For some $q \in\{1,2\}$, there exist a vertex $\mathbf{y}_{\mathbf{i}}$ and disjoint vertex subsets $\tilde{\mathbf{Y}} \subseteq \mathbf{Y}_{\mathbf{1}, \mathbf{q}}(\mathbf{Y}) \backslash\left\{\mathbf{y}_{\mathbf{i}}\right\}, \mathbf{Y}_{\mathbf{u}} \subseteq \mathbf{Y}_{\mathbf{1}, \mathbf{q}}(\mathbf{Y}) \backslash\left\{\mathbf{y}_{\mathbf{i}}\right\}, a$ $\mathbf{y}_{\mathbf{i}}$-topped path $P$ such that:

$\diamond \rho_{q}\left[\mathbf{U}_{\mathbf{1}, \mathbf{q}}(\mathbf{Y}) \cup \mathbf{X}_{\mathbf{s}, \mathbf{q}}(\mathbf{Y}), \mathbf{Y}_{\mathbf{u}}\right]=\operatorname{card}\left(\mathbf{U}_{\mathbf{1}, \mathbf{q}}(\mathbf{Y}) \cup \mathbf{X}_{\mathbf{s}, \mathbf{q}}(\mathbf{Y})\right)$,

$\diamond \beta_{q}\left(\tilde{\mathbf{Y}} \cup \mathbf{Y}_{\mathbf{u}}\right)+\beta_{q}\left(\left\{\mathbf{y}_{\mathbf{i}}\right\} \cup \mathbf{Y}_{\mathbf{u}}\right)-\beta_{q}\left(\tilde{\mathbf{Y}} \cup\left\{\mathbf{y}_{\mathbf{i}}\right\} \cup \mathbf{Y}_{\mathbf{u}}\right)>0$,

$\diamond \forall \mathbf{y}_{\mathbf{j}} \in \tilde{\mathbf{Y}}, \beta_{q}\left(\left(\tilde{\mathbf{Y}} \cup\left\{\mathbf{y}_{\mathbf{i}}\right\} \cup \mathbf{Y}_{\mathbf{u}}\right) \backslash\left\{\mathbf{y}_{\mathbf{j}}\right\}\right)-\beta_{q}\left(\mathbf{Y}_{\mathbf{u}} \cup \tilde{\mathbf{Y}} \backslash\left\{\mathbf{y}_{\mathbf{j}}\right\}\right)$ $>\beta_{q}\left(\tilde{\mathbf{Y}} \cup\left\{\mathbf{y}_{\mathbf{i}}\right\} \cup \mathbf{Y}_{\mathbf{u}}\right)-\beta_{q}\left(\tilde{\mathbf{Y}} \cup \mathbf{Y}_{\mathbf{u}}\right) \stackrel{\text { def }}{=} \widetilde{k_{i}}$,

$\diamond$ the length of $P$ is greater or equal to $\tilde{k}_{i}+1$

$\diamond \exists e \in \mathcal{E}_{q}$ belonging to a $S_{q}^{o}\left[\mathbf{v}_{\mathbf{P}}, \tilde{\mathbf{Y}} \cup\left\{\mathbf{y}_{\mathbf{i}}\right\}\right]-\tilde{\mathbf{Y}} \cup\left\{\mathbf{y}_{\mathbf{i}}\right\}$ path, where $\mathbf{v}_{\mathbf{P}}$ is the begin vertex of $P$.

\section{Proof:}

Sufficiency:

Condition Cond1.: Let us denote by $\mathbf{x}_{\mathbf{i}}$ one of the vertices of cycle $\mathcal{C}$ verifying Condition Cond1. Since this vertex belongs to $\mathbf{X}_{\mathbf{1}, \mathbf{q}}(\mathbf{Y})$, there exist a $\mathbf{x}_{\mathbf{i}}-\mathbf{y}_{\mathbf{j}}$ path in $\mathcal{E}_{0} \cup \mathcal{E}_{q}$, where $\mathbf{y}_{\mathbf{j}} \in \mathbf{Y}_{\mathbf{1}, \mathbf{q}}(\mathbf{Y})$, and a subset $\mathbf{Y}_{\mathbf{u}} \subset \mathbf{Y}_{\mathbf{1}, \mathbf{q}}(\mathbf{Y}) \backslash\left\{\mathbf{y}_{\mathbf{i}}\right\}$ with $\rho_{q}\left[\mathbf{U}_{\mathbf{1}, \mathbf{q}}(\mathbf{Y}) \cup \mathbf{X}_{\mathbf{s}, \mathbf{q}}(\mathbf{Y}), \mathbf{Y}_{\mathbf{u}}\right]=\operatorname{card}\left(\mathbf{U}_{\mathbf{1}, \mathbf{q}}(\mathbf{Y}) \cup\right.$ $\left.\mathbf{X}_{\mathbf{s}, \mathbf{q}}(\mathbf{Y})\right)<\rho_{q}\left[\mathbf{U}_{\mathbf{1}, \mathbf{q}}(\mathbf{Y}) \cup \mathbf{X}_{\mathbf{s}, \mathbf{q}}(\mathbf{Y}) \cup\left\{\mathbf{x}_{\mathbf{i}}\right\}, \mathbf{Y}_{\mathbf{u}} \cup\left\{\mathbf{y}_{\mathbf{j}}\right\}\right]$. From Lemma 2, there exist a matrix $G$, a function $\varphi$ and an integer $\nu \geq 0$ such that for mode $q,\left(X_{s, q}^{T}, U_{1, q}^{T}\right)^{T}=$ $\varphi\left(Y_{u}, \dot{Y}_{u}, \ldots, Y_{u}^{(\nu)}\right)+G X_{1, q}$. Substituting this in subsystem $\left(\Sigma_{1, q}\right)$ and using notations of (3):

$$
\left\{\begin{aligned}
\dot{X}_{1, q} & =\left(A_{1,1}+\left(A_{1, s}, B_{1,1}\right) G\right) X_{1, q}+\varphi_{x}\left(Y_{u}, \dot{Y}_{u}, \ldots, Y_{u}^{(\nu)}\right) \\
& \stackrel{\text { def }}{=} \tilde{A} X_{1, q}+\varphi_{x}\left(Y_{u}, \dot{Y}_{u}, \ldots, Y_{u}^{(\nu)}\right) \\
Y_{1, q} & =\left(C_{1,1}+\left(C_{1, s}, D_{1,1}\right) G\right) X_{1, q}+\varphi_{y}\left(Y_{u}, \dot{Y}_{u}, \ldots, Y_{u}^{(\nu)}\right) \\
& \stackrel{\text { def }}{=} \tilde{C} X_{1, q}+\varphi_{y}\left(Y_{u}, \dot{Y}_{u}, \ldots, Y_{u}^{(\nu)}\right)
\end{aligned}\right.
$$

Since the elements of $G$ are represented by the edges belonging to the $\mathbf{Y}_{\mathbf{u}}$-topped paths, they are independent from 
the elements of $A_{1,1}$ associated to cycle $\mathcal{C}$. Thus, the digraph representation of (4) contains also $\mathcal{C}$. Therefore, from (Reinschke, 1988) (Theorem 21.1), the characteristic equation of matrix $\tilde{A}$, which has the form $\tilde{A}^{n_{1}}+\ldots+a_{k} \tilde{A}^{k}+$ $\ldots+a_{0} \tilde{A}=0$, where $n_{1}=\operatorname{card}\left(\mathbf{X}_{\mathbf{1}, \mathbf{q}}(\mathbf{Y})\right)$, contains a term $a_{n_{1}-\bar{k}} \tilde{A}_{1,1}^{n_{1}-\bar{k}}$, where $\bar{k}$ is the length of $\mathcal{C}$ and $a_{n_{1}-\bar{k}}$ depends on the weight of $\mathcal{C}$ and so, on a specific entry of $A_{q}$ according to the existence of an edge belonging to $\mathcal{E}_{q}$ in $\mathcal{C}$. Thus,

$$
\left(\widetilde{C}_{j} \tilde{A}^{n_{1}}+\ldots+a_{k} \tilde{C}_{j} \tilde{A}^{k}+\ldots+a_{0} \tilde{C}_{j} \tilde{A}\right) X_{1, q}=0
$$

where $\tilde{C}_{j}$ is the line of matrix $\widetilde{C}$ related to output $y_{j}$ in mode $q$ in (4) i.e. $y_{j}=\tilde{C}_{j} X_{1, q}+\varphi_{y, j}\left(Y_{u}, \dot{Y}_{u}, \ldots, Y_{u}^{(\nu)}\right)$. On the one hand, due to dynamics (4) for all $k \geq 0$ $\tilde{C}_{j} \tilde{A}^{k}=y_{j}^{(k)}+\varphi_{u}\left(Y_{u}, \dot{Y}_{u}, \ldots, Y_{u}^{(\nu+k)}\right)$. On the other hand, since there exists a path from $\mathbf{x}_{\mathbf{i}}$ to $\mathbf{y}_{\mathbf{j}}$, if we denote by $\ell>0$ the length of this path, then $\forall k \geq \ell-1, \tilde{C}_{j} \tilde{A}^{k} \neq 0$. Furthermore, since $\ell+\bar{k} \leq n_{1}$, then term $a_{n_{1}-\bar{k}} \tilde{A}_{1,1}^{n_{1}-\bar{k}} \neq 0$. Therefore, equation (5) leads to an algebraic equation of the kind $\Psi_{q}\left(Y_{1, q}, \dot{Y}_{1, q}, \ldots, Y_{1, q}^{(s)}\right)=0$ parameterized by at least an element of $A_{q}$ specific to mode $q$.

Condition Cond2.: Let denote by $\mathbf{v}_{\mathbf{i}}$ the vertex of $\mathbf{U}_{\mathbf{1}, \mathbf{q}}(\mathbf{Y}) \cup \mathbf{X}_{\mathbf{s}, \mathbf{q}}(\mathbf{Y})$ from which there is a direct $\mathbf{X}_{\mathbf{s}, \mathbf{q}}(\mathbf{Y}) \cup \mathbf{U}_{\mathbf{1}, \mathbf{q}}(\mathbf{Y})-\mathbf{Y}_{\mathbf{1}, \mathbf{q}}(\mathbf{Y})$ path $P$ included in $\mathcal{E}_{0} \cup \mathcal{E}_{q}$ and containing at least an edge $e_{\kappa}$ of $\mathcal{E}_{q}$. Let us denote by $\lambda_{\kappa}$ the non-zero parameter (or weight) associated to $e_{\kappa}$. Let $\mathbf{y}_{\mathbf{j}}$ be the end of $P$ and $\ell$ its length. Since $\mathbf{v}_{\mathbf{i}}$ belongs to $\mathbf{U}_{\mathbf{1 , q}}(\mathbf{Y}) \cup \mathbf{X}_{\mathbf{s}, \mathbf{q}}(\mathbf{Y})$, it exists a subset $\mathbf{Y}_{\mathbf{u}}=\left\{\mathbf{y}_{\mathbf{i}_{\mathbf{1}}}, \mathbf{y}_{\mathbf{i}_{\mathbf{2}}}, \ldots, \mathbf{y}_{\mathbf{i}_{\mathbf{k}}}\right\} \subset \mathbf{Y}_{\mathbf{1}, \mathbf{q}}(\mathbf{Y}) \backslash\left\{\mathbf{y}_{\mathbf{i}}\right\}$ such that $\rho_{q}\left[\mathbf{U}_{\mathbf{1}, \mathbf{q}}(\mathbf{Y}) \cup \mathbf{X}_{\mathbf{s}, \mathbf{q}}(\mathbf{Y}), \mathbf{Y}_{\mathbf{u}}\right]=\operatorname{card}\left(\mathbf{U}_{\mathbf{1}, \mathbf{q}}(\mathbf{Y}) \cup \mathbf{X}_{\mathbf{s}, \mathbf{q}}(\mathbf{Y})\right)$ without using edges and vertices of $P$. From Lemma 2, there exist a matrix $G$, a function $\varphi$ and an integer $\nu \leq n_{1}$ such that the dynamics equation of subsystem $\left(\Sigma_{1, q}\right)$ is in the form (4). Since the elements of $G$ are represented by edges belonging to the $\mathbf{Y}_{\mathbf{u}}$-topped paths, they are independent from the elements of $A_{1,1}$. From the characteristic equation of matrix $\tilde{A}$, we can write an equation as (5), where also $\tilde{C}_{j}$ is the line of matrix $\widetilde{C}$ related to output $y_{j}$ in mode $q$ in (4) i.e. $y_{j}=\tilde{C}_{j} X_{1, q}+\varphi_{y, j}\left(Y_{u}, \dot{Y}_{u}, \ldots, Y_{u}^{(\nu)}\right)$ and so, $\tilde{C}_{j} \tilde{A}_{1,1}^{k} X_{1, q}=y_{j}^{(k)}-\tilde{C}_{j}\left(\varphi_{x}^{(k-1)}\left(Y_{u}, \dot{Y}_{u}, \ldots, Y_{u}{ }^{(\nu)}\right)+\right.$ $\tilde{A}_{1,1} \varphi_{x}^{(k-2)}\left(Y_{u}, \dot{Y}_{u}, \ldots, Y_{u}^{(\nu)}\right) \quad+\quad \ldots \quad+$ $\left.\tilde{A}_{1,1}^{k-2} \dot{\varphi}_{x}\left(Y_{u}, \dot{Y}_{u}, \ldots, Y_{u}^{(\nu)}\right)\right)-\varphi_{y, j}^{(k)}\left(Y_{u}, \dot{Y}_{u}, \ldots, Y_{u}^{(\nu)}\right)$. Since there exists a path of length $\ell$ from $\mathbf{v}_{\mathbf{i}}$ to $\mathbf{y}_{\mathbf{j}}$ containing an edge $e_{\kappa}$, term $C_{j} \tilde{A}_{1,1}^{\ell-1} \varphi_{x}^{(k-\ell)}\left(Y_{u}, \dot{Y}_{u}, \ldots, Y_{u}^{(\nu)}\right)$, for all $k \geq \ell$, is not zero and depends on the element of $A_{q}$ denoted previously $\lambda_{\kappa}$ which is specific to location $q$. In particular, first term $\tilde{C}_{j} \tilde{A}^{n_{1}}$, which is present in relation (5) is not zero and depends on $\lambda_{\kappa}$. Thus, equation (5) leads to an algebraic equation $\Psi_{q}\left(Y, \dot{Y}, \ldots, Y^{(s)}\right)=0$ parameterized by at least an element of $A_{q}$.

Condition Cond3.: First, $\mathbf{y}_{\mathbf{i}} \in \mathbf{Y}_{\mathbf{1}, \mathbf{q}}(\mathbf{Y}) \backslash \mathbf{Y}_{\mathbf{1}, \mathbf{q}^{\prime}}(\mathbf{Y})$ means that there is a redundancy equation linking $y_{i}, Y_{u}$ and their derivatives in mode $q$ but not in mode $q^{\prime}$ which achieves location observability.

Otherwise, when $\mathbf{y}_{\mathbf{i}} \in \mathbf{Y}_{\mathbf{1}, \mathbf{q}}(\mathbf{Y}) \cap \mathbf{Y}_{\mathbf{1}, \mathbf{q}^{\prime}}(\mathbf{Y})$, let us introduce the following notation $k_{i, 1} \stackrel{\text { def }}{=} \beta_{1}\left(\tilde{\mathbf{Y}} \cup\left\{\mathbf{y}_{\mathbf{i}}\right\} \cup \mathbf{Y}_{\mathbf{u}}\right)-$ $\beta_{1}\left(\tilde{\mathbf{Y}} \cup \mathbf{Y}_{\mathbf{u}}\right)$ and $k_{i, 2} \stackrel{\text { def }}{=} \beta_{2}\left(\tilde{\mathbf{Y}} \cup\left\{\mathbf{y}_{\mathbf{i}}\right\} \cup \mathbf{Y}_{\mathbf{u}}\right)-\beta_{2}\left(\tilde{\mathbf{Y}} \cup \mathbf{Y}_{\mathbf{u}}\right)$.
According to Lemma 3, this implies that output $y_{i}$ allows to observe $k_{i_{1}}$ (resp. $k_{i_{1}}$ ) new directions w.r.t. $\mathbf{Y}_{\mathbf{u}}$ and $\tilde{\mathbf{Y}}$ in the extended state and input space for mode $q$ (resp. $q^{\prime}$ ). Therefore, we have that $k_{i, 1}$ (resp. $k_{i, 2}$ ) is the minimal integer such that there exists an expression of $y_{i}^{\left(k_{i, 1}\right)}$ (resp. $y_{i}^{\left(k_{i, 1}\right)}$ ), satisfied in mode 1 (resp. 2), depending on the first $k_{i, 1}-1$ (resp. $k_{i, 2}-1$ ) derivatives of $y_{i}, Y_{u}$ and $\tilde{Y}$ and their derivatives i.e. $\exists s_{1} \geq 0$ (resp. $\left.\exists s_{2} \geq 0\right)$ and function $\varphi_{1}\left(\operatorname{resp} \varphi_{2}\right)$ such that $y_{i}^{\left(k_{i, 1}\right)}=$ $\varphi_{1}\left(y_{i}, \dot{y}_{i}, \ldots, y_{i}^{\left(k_{i, 1}-1\right)}, Y_{u}, \ldots, Y_{u}^{\left(s_{1}\right)}, \tilde{Y}, \ldots, \tilde{Y}^{\left(s_{1}\right)}\right)$ (resp. $y_{i}^{\left(k_{i, 2}\right)}=\varphi_{2}\left(y_{i}, \ldots, y_{i}^{\left(k_{i, 2}-1\right)}, Y_{u}, \ldots, Y_{u}^{\left(s_{2}\right)}, \tilde{Y}\right.$, $\left.\ldots, \tilde{Y}^{\left(s_{2}\right)}\right)$ ). Consequently, when $k_{i, 1} \neq k_{i, 2}$, one of these two numbers is strictly lower than the other. If $k_{i, 1}<k_{i, 2}$, then expression $y_{i}^{\left(k_{i, 1}\right)}=$ $\varphi_{1}\left(y_{i}, \ldots, y_{i}^{\left(k_{i, 1}-1\right)}, Y_{u}, \dot{Y}_{u}, \ldots, Y_{u}^{\left(s_{1}\right)}, \tilde{Y}, \dot{\tilde{Y}}, \ldots, \tilde{Y}^{\left(s_{1}\right)}\right)$ satisfied in mode 1 cannot be valid in mode 2 . When $k_{i, 2}<k_{i, 1}$, we can do similar reasoning. In both the cases, we obtain an expression, which allows to distinguish the modes.

Condition Cond4.: The fact that for some $q$ there exists a vertex subset $\mathbf{Y}_{\mathbf{u}} \subseteq \mathbf{Y}_{\mathbf{1}, \mathbf{q}}(\mathbf{Y}) \backslash\left\{\mathbf{y}_{\mathbf{i}}\right\}$ such that $\rho_{q}\left[\mathbf{U}_{\mathbf{1}, \mathbf{q}}(\mathbf{Y}) \cup \mathbf{X}_{\mathbf{s}, \mathbf{q}}(\mathbf{Y}), \mathbf{Y}_{\mathbf{u}}\right]=\operatorname{card}\left(\mathbf{U}_{\mathbf{1}, \mathbf{q}}(\mathbf{Y}) \cup \mathbf{X}_{\mathbf{s}, \mathbf{q}}(\mathbf{Y})\right)$ implies, from Lemma 2 , that there exist a matrix $G$, a function $\varphi$ and an integer $\nu \leq n_{1}$ such that the dynamics equation of subsystem $\left(\Sigma_{1, q}\right)$ can be put on form (4). Moreover,the existence of a vertex $y_{i}$ and vertex subset $\tilde{\mathbf{Y}} \subseteq \mathbf{Y}_{\mathbf{1}, \mathbf{q}}(\mathbf{Y}) \backslash\left\{\mathbf{y}_{\mathbf{i}}\right\}$ disjoint with $\mathbf{Y}_{\mathbf{u}}$ such that $\beta_{q}\left(\tilde{\mathbf{Y}} \cup \mathbf{Y}_{\mathbf{u}}\right)+\beta_{q}\left(\left\{\mathbf{y}_{\mathbf{i}}\right\} \cup \mathbf{Y}_{\mathbf{u}}\right)-\beta_{q}\left(\left(\tilde{\mathbf{Y}} \cup\left\{\mathbf{y}_{\mathbf{i}}\right\} \cup \mathbf{Y}_{\mathbf{u}}\right)>0\right.$ implies that, for mode $q$, denoting by $\beta_{q}\left(\tilde{\mathbf{Y}} \cup\left\{\mathbf{y}_{\mathbf{i}}\right\} \cup \mathbf{Y}_{\mathbf{u}}\right)-$ $\beta_{q}\left(\tilde{\mathbf{Y}} \cup \mathbf{Y}_{\mathbf{u}}\right) \stackrel{\text { def }}{=} \widetilde{k_{i}}$, we have $\forall k \geq \tilde{k}_{i}$,

$$
y_{i}^{(k)}=\sum_{s<\tilde{k_{i}}} \alpha_{i, s} y_{i}^{(s)}+\sum_{l \mid \mathbf{y}_{l} \in \tilde{\mathbf{Y}}} \sum_{s=0}^{n_{1}} \alpha_{l, s} y_{l}^{(s)}+v\left(Y_{u}, \ldots, Y_{u}^{\left(n_{1}\right)}\right)
$$

where $n_{1}=\operatorname{card}\left(\mathbf{X}_{\mathbf{1}, \mathbf{q}}\right)$. Since subset $\tilde{\mathbf{Y}}$ is minimal i.e. $\left.\forall \mathbf{y}_{\mathbf{j}} \in \tilde{\mathbf{Y}}, \beta_{q}\left(\tilde{\mathbf{Y}} \cup\left\{\mathbf{y}_{\mathbf{i}}\right\} \cup \mathbf{Y}_{\mathbf{u}}\right) \backslash\left\{\mathbf{y}_{\mathbf{j}}\right\}\right)-\beta_{q}\left(\mathbf{Y}_{\mathbf{u}} \cup \tilde{\mathbf{Y}} \backslash\right.$ $\left.\left\{\mathbf{y}_{\mathbf{j}}\right\}\right)>\widetilde{k_{i}}$, then in relation (6), all the components of $\tilde{\mathbf{Y}}$ intervene. Let us denote by $\mathbf{v}_{\mathbf{P}}=\mathbf{x}_{\mathbf{j}}$ the begin vertex of path $P$ satisfying Condition Cond4. and $\mathrm{e}_{j}$ the $j^{\text {th }}$ Euclidean vector. Relation (6), where $k+1$ is here the length of $P$ can be written as:

$\tilde{C}_{i} \widetilde{A}^{k} \mathrm{e}_{j}=\left(\sum_{s<\tilde{k_{i}}} \alpha_{i, s} \tilde{C}_{i} \widetilde{A}^{s}+\sum_{\mathbf{y}_{l} \in \tilde{\mathbf{Y}}} \sum_{s=0}^{n_{1}} \alpha_{l, s} \tilde{C}_{l} \tilde{A}^{s}+v\left(Y_{u}, \ldots, Y_{u}^{\left(n_{1}\right)}\right)\right) \mathrm{e}_{j}$

where each non-zero component of $\tilde{C}_{l} \tilde{A}^{s}$ is associated to the paths arriving to $\mathbf{y}_{l} \in \tilde{Y}$ of length $s+1$. Since all the $\left\{\mathbf{x}_{\mathbf{j}}\right\}-\tilde{\mathbf{Y}} \cup\left\{\mathbf{y}_{\mathbf{i}}\right\}$ paths starting from $\mathbf{x}_{\mathbf{j}}$ cover, by definition, $S_{q}^{o}\left[\left\{\mathbf{x}_{\mathbf{j}}\right\}, \tilde{\mathbf{Y}} \cup\left\{\mathbf{y}_{\mathbf{i}}\right\}\right] \stackrel{\text { def }}{=}\left\{\mathbf{x}_{\mathbf{r}}\right\}$, then there exist $k_{r}$ and $k^{\prime}$ such that $k_{r}+k^{\prime}=k$ and $\tilde{C}_{i} \tilde{A}^{k} \mathrm{e}_{j}=\tilde{C}_{i} A^{k_{r}} \Delta_{r} \tilde{A}^{k^{\prime}} \mathrm{e}_{j}$ where $\Delta_{r}$ is a diagonal matrix which has only one non-zero element $\Delta_{r}(r, r)=1$. We can do the same reasoning for each term $\tilde{C}_{l} \tilde{A}^{s} \mathrm{e}_{j}$ and so there exist $s_{r}$ and $s^{\prime}$ such that $s_{r}^{\prime}+s^{\prime}=s$ and $\tilde{C}_{l} \tilde{A}^{s} \mathrm{e}_{j}=\tilde{C}_{l} \tilde{A}^{s_{r}} \Delta_{r} \tilde{A}^{s^{\prime}} \mathrm{e}_{j}$. The fact that there is an edge $e_{\kappa} \in \mathcal{E}_{q}$ belonging to a $S_{q}^{o}\left[\mathbf{v}_{\mathbf{P}}, \tilde{\mathbf{Y}} \cup\left\{\mathbf{y}_{\mathbf{i}}\right\}\right]-$ $\tilde{\mathbf{Y}} \cup\left\{\mathbf{y}_{\mathbf{i}}\right\}$ path means that this edge $e_{\kappa}$ appears in only some of $S_{q}^{o}\left[\mathbf{v}_{\mathbf{P}}, \tilde{\mathbf{Y}} \cup\left\{\mathbf{y}_{\mathbf{i}}\right\}\right]-\tilde{\mathbf{Y}} \cup\left\{\mathbf{y}_{\mathbf{i}}\right\}$ paths. Thus, some, but not 
all, terms $\tilde{C}_{i} A^{k_{r}}$ and $\tilde{C}_{l} \tilde{A}^{s_{r}}$ contain the non-zero parameter corresponding to edge $e_{\kappa}$, which is specific to mode $q$ of SSLS $\left(\Sigma_{\Lambda}\right)$. Denoting by $C_{r}=\mathrm{e}_{r}^{T}$, where $\mathrm{e}_{r}$ is the $r^{t h}$ Euclidean vector, we have that $\tilde{C}_{i} \tilde{A}^{k} \mathrm{e}_{j}=\tilde{C}_{i} A^{k_{r}} \Delta_{r} \tilde{A}^{k^{\prime}} \mathrm{e}_{j}=$ $\alpha^{\prime} C_{r} \tilde{A}^{k^{\prime}} \mathrm{e}_{j}$ and $\tilde{C}_{l} \tilde{A}^{s} \mathrm{e}_{j}=\tilde{C}_{l} \tilde{A}^{s_{r}} \Delta_{r} \tilde{A}^{s^{\prime}} \mathrm{e}_{j}=\alpha_{l, s}^{\prime} C_{r} \tilde{A}^{s^{\prime}} \mathrm{e}_{j}$. Thus, after substitution of the previous terms in relation (7),

$$
\begin{aligned}
& \alpha^{\prime} C_{r} \widetilde{A}^{k^{\prime}} \mathrm{e}_{j}=\left(\sum_{s_{r} \leq s<\tilde{k}_{i}} \alpha_{i, s}^{\prime} \alpha_{i, s} C_{r} \widetilde{A}^{s-s_{r}}+\right. \\
& \left.\sum_{l \mid \mathbf{y}_{l} \in \tilde{\mathbf{Y}}} \sum_{s=s_{r}}^{n_{1}} \alpha_{l, s}^{\prime} \alpha_{l, s} C_{r} \tilde{A}^{s-s_{r}}+v\left(Y_{u}, \dot{Y}_{u}, \ldots, Y_{u}^{(n)}\right)\right) \mathrm{e}_{j}
\end{aligned}
$$

where some, but not all coefficients $\alpha^{\prime}$ and $\alpha_{l, s}^{\prime}$ depend on the weight of $e_{\kappa}$. This weight cannot be factorized and simplified because all the coefficients do not depend on it (some $S_{q}^{o}\left[\mathbf{v}_{\mathbf{P}}, \tilde{\mathbf{Y}} \cup\left\{\mathbf{y}_{\mathbf{i}}\right\}\right]-\tilde{\mathbf{Y}} \cup\left\{\mathbf{y}_{\mathbf{i}}\right\}$ paths do not contain edge $e_{\kappa}$ ). Therefore, equality (8) is valid only if some of the coefficients $\alpha, \alpha_{i, s}$ and $\alpha_{l, s}$ depend also on the weight $\lambda_{\kappa}$ of $e_{\kappa}$. Thus, by means of equation (6) in which appear coefficients $\alpha_{i, s}$ and $\alpha_{l, s}$, we obtain an algebraic relation depending on $\lambda_{\kappa}$ and satisfied only when the discrete mode variable is equal to $q$.

Necessity: Firstly, from Lemma 1, there cannot exist an algebraic equation linking the output components of subsystem $\left(\Sigma_{0, q}\right)$. So, only the edges in subsystem $\left(\Sigma_{1, q}\right)$ can lead to an algebraic relation between outputs $\mathbf{Y}_{\mathbf{1 , q}}(\mathbf{Y})$ and their derivatives in order to deduce the discrete mode. In this case, some outputs have to be used to invert $\left(\Sigma_{1, q}\right)$ in order to eliminate from the output equations all the unknown inputs and so to use all the possible derivatives of the other outputs in a redundancy equation. It is necessary then to consider a subset $\mathbf{Y}_{\mathbf{u}} \subseteq \mathbf{Y}_{\mathbf{1}, \mathbf{q}}(\mathbf{Y})$ such that $\rho_{q}\left[\mathbf{U}_{\mathbf{1}, \mathbf{q}}(\mathbf{Y}) \cup \mathbf{X}_{\mathbf{s}, \mathbf{q}}(\mathbf{Y}), \mathbf{Y}_{\mathbf{u}}\right]=\operatorname{card}\left(\mathbf{U}_{\mathbf{1}, \mathbf{q}}(\mathbf{Y}) \cup \mathbf{X}_{\mathbf{s}, \mathbf{q}}(\mathbf{Y})\right)$. Indeed, from Lemma 2 and Theorem 4 of (Dion et $a l ., 2003)$, only in such a case the system is left-invertible and so there exist integer $\nu \geq 0$, matrix $G$ and function $\varphi$ such that $\left(X_{s, q}^{T}, U_{1, q}^{T}\right)^{T}=\varphi\left(Y_{u}, \dot{Y}_{u}, \ldots, Y_{u}^{(\nu)}\right)+G X_{1, q}$, which can be substituted in subsystem $\left(\Sigma_{1, q}\right)$ for mode $q$, to obtain an equation of form (4). When Conditions Cond1. and Cond2. are not satisfied, it is not possible to obtain an algebraic relation achieving mode distinguishability from the characteristic equation of any matrix $\tilde{A}$ of (4) related to any choice of $\mathrm{Y}_{\mathrm{u}}$, when this equation has the same degree for the two modes. It is the case when Cond3. is not satisfied. Moreover, when condition Cond2. is not satisfied, $\varphi_{y}\left(Y_{u}, \dot{Y}_{u}, \ldots, Y_{u}^{(\nu)}\right)$ and $\varphi_{x}\left(Y_{u}, \dot{Y}_{u}, \ldots, Y_{u}^{(\nu)}\right)$ do not depend on any specific element of $A_{q}, B_{q}, C_{q}$ or $D_{q}$, $q \in\{1,2\}$. Furthermore, any output equation can be put on the form (6), for some integer $k$, output component $\mathbf{y}_{\mathbf{i}}$ and some set of output components $\widetilde{\mathbf{Y}}$ which do not include $\mathbf{y}_{\mathbf{i}}$ and are disjoint from $\mathbf{Y}_{\mathbf{u}}$. Moreover, when Condition Cond3. is not satisfied, for any of these expressions (when they exist), the degree $k$ is the same for modes 1 and 2 . Thus, the only way to have a difference between the two algebraic equations respectively is that there exists at least one coefficient $\alpha_{i, s}$ or $\alpha_{l, s}$ of 6 , which characterizes specifically a parameter which intervenes in only one of the two modes' model. Without loss of generality, we can assume that $\widetilde{\mathbf{Y}}$ is minimal i.e. in the algebraic relations and so all the components of $\tilde{\mathbf{Y}}$ appear. If it is not the case, we remove them from $\widetilde{\mathbf{Y}}$. Relation (6) implies that the observable subspace obtained using outputs $\left\{\mathbf{y}_{\mathbf{i}}\right\} \cup \mathbf{Y}_{\mathbf{u}}$ and the one generated by $\tilde{\mathbf{Y}} \cup \mathbf{Y}_{\mathbf{u}}$ have non zero intersection. Thus, an algebraic equation linking some output components of $\left(\Sigma_{1, q}\right)$ is possible in mode $q$ only if there exist a vertex $\mathbf{y}_{\mathbf{i}}$ and disjoint vertex subsets $\tilde{\mathbf{Y}} \subseteq \mathbf{Y}_{\mathbf{1}, \mathbf{q}}(\mathbf{Y}) \backslash\left\{\mathbf{y}_{\mathbf{i}}\right\}, \mathbf{Y}_{\mathbf{u}} \subseteq \mathbf{Y}_{\mathbf{1}, \mathbf{q}}(\mathbf{Y}) \backslash\left\{\mathbf{y}_{\mathbf{i}}\right\}$, with $\rho_{q}\left[\mathbf{U}_{\mathbf{1}, \mathbf{q}}(\mathbf{Y}) \cup \mathbf{X}_{\mathbf{s}, \mathbf{q}}(\mathbf{Y}), \mathbf{Y}_{\mathbf{u}}\right]=\operatorname{card}\left(\mathbf{U}_{\mathbf{1}, \mathbf{q}}(\mathbf{Y}) \cup \mathbf{X}_{\mathbf{s}, \mathbf{q}}(\mathbf{Y})\right)$, and such that $\beta_{q}\left(\tilde{\mathbf{Y}} \cup \mathbf{Y}_{\mathbf{u}}\right)+\beta_{q}\left(\left\{\mathbf{y}_{\mathbf{i}}\right\} \cup \mathbf{Y}_{\mathbf{u}}\right)-\beta_{q}(\tilde{\mathbf{Y}} \cup$ $\left.\left\{\mathbf{y}_{\mathbf{i}}\right\} \cup \mathbf{Y}_{\mathbf{u}}\right)>0$. Assume that this inequality is satisfied and let us specify the necessary condition which guarantees that the concerned algebraic equation allows us to deduce the current mode of the system. Defining $\widetilde{k}_{i}=\beta_{q}\left(\tilde{\mathbf{Y}} \cup\left\{\mathbf{y}_{\mathbf{i}}\right\} \cup \mathbf{Y}_{\mathbf{u}}\right)-\beta_{q}\left(\tilde{\mathbf{Y}} \cup \mathbf{Y}_{\mathbf{u}}\right)$, relation (6) cannot be satisfied for some $k \leq \widetilde{k}_{i}-1$. Thus, in redundancy relation (6), $k \geq \widetilde{k_{i}}$. Furthermore, since $\widetilde{\mathbf{Y}}$ is minimal, we cannot express $y_{i}^{\left(\tilde{k}_{i}\right)}$ using only a part of $\tilde{Y}$ i.e. $\forall \mathbf{y}_{\mathbf{j}} \in \tilde{\mathbf{Y}}$, $\beta_{q}\left(\left(\tilde{\mathbf{Y}} \cup \mathbf{Y}_{\mathbf{u}} \cup\left\{y_{i}\right\}\right) \backslash\left\{\mathbf{y}_{\mathbf{j}}\right\}\right)-\beta_{q}\left(\mathbf{Y}_{\mathbf{u}} \cup \tilde{\mathbf{Y}} \backslash\left\{\mathbf{y}_{\mathbf{j}}\right\}\right)>\widetilde{k}_{i}$. To guarantee location observability, there must exist at least a $\mathbf{y}_{\mathbf{i}}$-topped path $P$, of length greater or equal to $\widetilde{k_{i}}+1$, associated to this relation. Let us denote by $\mathbf{x}_{\mathbf{j}}$ (it cannot be a $\mathbf{U}_{\mathbf{1}, \mathbf{q}}(\mathbf{Y}) \cup \mathbf{X}_{\mathbf{s}, \mathbf{q}}(\mathbf{Y})-\mathbf{Y}_{\mathbf{1}, \mathbf{q}}(\mathbf{Y})$ path when $\mathbf{C o n d 2}$. is not satisfied) the begin vertex of $P$ and $\mathrm{e}_{j}$ the $j^{\text {th }}$ Euclidean vector. Moreover, when condition Cond2. is not satisfied, in Equation (6), $v\left(Y_{u}, \dot{Y}_{u}, \ldots, Y_{u}^{(n)}\right)$ does not depend on any element specific of $A_{q}, B_{q}, C_{q}$ or $D_{q}(q \in\{1,2\})$. Then, equation (6), can be written as relation (7), where $\tilde{C}_{l} \tilde{A}^{s}$ is associated $\mathbf{y}_{l}$-topped paths, $\mathbf{y}_{l} \in \tilde{\mathbf{Y}}$ of length $s+1$. Since all the $\left\{\mathbf{x}_{\mathbf{j}}\right\}-\tilde{\mathbf{Y}} \cup\left\{\mathbf{y}_{\mathbf{i}}\right\}$ paths cover $\mathbf{x}_{\mathbf{r}}=S_{q}^{o}\left[\mathbf{x}_{\mathbf{j}}, \tilde{\mathbf{Y}} \cup\left\{\mathbf{y}_{\mathbf{i}}\right\}\right]$, then according to the same arguments as previously (in the part of the proof devoted to the sufficiency of condition Cond4.), we can write an equation similar to (8) with the same notations, where $\alpha^{\prime}$ and $\alpha_{l, s^{\prime}}^{\prime}$ depend only on the entries related to the edges of $\mathcal{E}_{0}$ when Cond4. is not satisfied. So, all the existing relations of the form (6) do not contain terms of $A_{q}$, $B_{q}, C_{q}$ or $D_{q}(q \in\{1,2\})$. We can do the same reasoning for all $\mathbf{Y}_{\mathbf{u}} \subseteq \mathbf{Y}_{\mathbf{1}, \mathbf{q}}(\mathbf{Y})$ s.t. $\rho_{q}\left[\mathbf{U}_{\mathbf{1}, \mathbf{q}}(\mathbf{Y}) \cup \mathbf{X}_{\mathbf{s}, \mathbf{q}}(\mathbf{Y}), \mathbf{Y}_{\mathbf{u}}\right]$ $=\operatorname{card}\left(\mathbf{U}_{\mathbf{1}, \mathbf{q}}(\mathbf{Y}) \cup \mathbf{X}_{\mathbf{s}, \mathbf{q}}(\mathbf{Y})\right)$.

Comments and interpretation: To establish location observability, we first search in the graph the edges which are specific to one mode and we interest to their position w.r.t. the output vertices. If there is a specific edge belonging to any cycle in $\left(\Sigma_{1, q}\right)$ then the distinguishability is possible (first condition). If a specific edge belong to any input-output path in $\left(\Sigma_{1, q}\right)$ then the distinguishability is possible (second condition). If a specific edge allows to modify observability subspace of any output measurements set or to modify the output subdivision $\mathbf{Y}_{\mathbf{0}} / \mathbf{Y}_{\mathbf{1}}$, then the distinguishability is possible (third condition). Finally, if a specific edge belong to an output rooted path including a state vertex which can be linked independently to other output, with some constraints on observability subspaces' dimensions, then the distinguishability is possible (fourth condition). If all the conditions are not satisfied, then the two modes are sufficiently similar or their differences are not observable from the measurements.

Note that, the second condition of Proposition 2 generalizes the condition (De Santis et al., 2006; De Santis et al., 2009) of location observability for non autonomous systems, where the inputs are measured $(\exists i \geq 0$ such that $\left.C(1) A^{i}(1) B(1) \neq C(2) A^{i}(2) B(2)\right)$. The first two conditions, which can be checked immediately by searching cycles and $\mathbf{X}_{\mathbf{s}, \mathbf{q}}(\mathbf{Y}) \cup \mathbf{U}_{\mathbf{1}, \mathbf{q}}(\mathbf{Y})-\mathbf{Y}_{\mathbf{1}, \mathbf{q}}(\mathbf{Y})$ paths, are based on the characteristic equation of a matrix. So, both the conditions of Definition 1, are satisfied. This means, using definition of (Babaali and Pappas, 2005), that modes $q$ and 
$q^{\prime}$ are mutually discernible. It is the same when Condition Cond4. is satisfied for some subsets $\mathbf{Y}_{\mathbf{u}}, \tilde{\mathbf{Y}}$ and $\left\{\mathbf{y}_{\mathbf{i}}\right\}$, which do not satisfy Condition Cond3. because, in this case, there exist two expressions linking these output components and their derivatives. One of them specific to some mode $q$ means that the other is specific to mode $q^{\prime}$. In such a case also both the conditions of Definition 1 are satisfied and we obtain the mutual mode discernibility property as defined in (Babaali and Pappas, 2005). Finally, when only Condition Cond3. is satisfied, then there exists only one discriminating expression. In fact, if $\beta_{q}\left(\tilde{\mathbf{Y}} \cup\left\{\mathbf{y}_{\mathbf{i}}\right\} \cup \mathbf{Y}_{\mathbf{u}}\right)-\beta_{q}\left(\tilde{\mathbf{Y}} \cup \mathbf{Y}_{\mathbf{u}}\right)<$ $\beta_{q^{\prime}}\left(\tilde{\mathbf{Y}} \cup\left\{\mathbf{y}_{\mathbf{i}}\right\} \cup \mathbf{Y}_{\mathbf{u}}\right)-\beta_{q^{\prime}}\left(\tilde{\mathbf{Y}} \cup \mathbf{Y}_{\mathbf{u}}\right)$ then expression specific to mode $q$ is not satisfied by mode $q^{\prime}$ but the expression obtained for mode $q^{\prime}$, which is of a greater degree, is satisfied by mode $q$. If only Condition Cond3. is satisfied and if, for each $\mathbf{Y}_{\mathbf{u}}, \tilde{\mathbf{Y}}$ and $\mathbf{y}_{\mathbf{i}}$ satisfying Cond3., we have the same inequality sense between modes $q$ and $q^{\prime}$, then only one condition of Definition 1 is verified. According to definition of (Babaali and Pappas, 2005), $q$ is not discernible from $q^{\prime}$. To summarize, the conditions enounced in Proposition 2 can be classified into two categories. The first one includes conditions Cond3., a part of Cond1. and Cond2. when the subdivision of the system for the two modes are different. In this case, the two modes can be said structurally distinguishable. The distnguishability here depends on the structure of the systems associated to each mode and not on the parameters' values. In the case of condition Cond1., when the cycle exists in a mode and not in the other one, there is then at least an additional relation differentiating the two modes and so the distinguishability is here also structural. In all the other cases, the distinguishability is parametric.

Exemple 1 Continued Let us see if it is possible to find specific equation characterizing mode 1 . We illustrate the computations of condition Cond4., which is the more complicated. It is easy to see that conditions Cond1. and Cond2. are not satisfied. There is only one edge $\left(\mathbf{x}_{2}, \mathbf{x}_{1}\right)$ which is specific to mode 1 . Since $\mathbf{X}_{\mathbf{s}, \mathbf{1}}=\left\{\mathbf{x}_{\mathbf{8}}\right\}$, we can take for $\mathbf{Y}_{\mathbf{u}}$ one of the outputs $\mathbf{y}_{\mathbf{3}}$ or $\mathbf{y}_{\mathbf{4}}$. For instance, let us take $\mathbf{Y}_{\mathbf{u}}=\left\{\mathbf{y}_{\mathbf{4}}\right\}, \mathbf{y}_{\mathbf{i}}=\mathbf{y}_{\mathbf{1}}$ and for example $\tilde{\mathbf{Y}}=\left\{\mathbf{y}_{\mathbf{2}}\right\}$. In this case, we have that:

$\diamond \rho_{1}\left[\mathbf{U}_{\mathbf{1}, \mathbf{1}}(\mathbf{Y}) \cup \mathbf{X}_{\mathbf{s}, \mathbf{1}}(\mathbf{Y}), \mathbf{Y}_{\mathbf{u}}\right]=1, \beta_{1}\left(\tilde{\mathbf{Y}} \cup \mathbf{Y}_{\mathbf{u}}\right)=7, \beta_{1}(\tilde{\mathbf{Y}} \cup$ $\left.\left\{\mathbf{y}_{\mathbf{1}}\right\}\right)=7$ and $\beta_{1}\left(\tilde{\mathbf{Y}} \cup\left\{\mathbf{y}_{\mathbf{1}}\right\} \cup \mathbf{Y}_{\mathbf{u}}\right)=8$. So, $\beta_{1}\left(\tilde{\mathbf{Y}} \cup \mathbf{Y}_{\mathbf{u}}\right)+$ $\beta_{1}\left(\left\{\mathbf{y}_{\mathbf{1}}\right\} \cup \mathbf{Y}_{\mathbf{u}}\right)-\beta_{1}\left(\tilde{\mathbf{Y}} \cup\left\{\mathbf{y}_{\mathbf{1}}\right\} \cup \mathbf{Y}_{\mathbf{u}}\right)=6>0$. Since $\tilde{\mathbf{Y}}$ is constituted of only one output, it is then minimal and obviously, $\forall \mathbf{y}_{\mathbf{j}} \in \tilde{\mathbf{Y}}, \beta_{1}\left(\left(\tilde{\mathbf{Y}} \cup\left\{\mathbf{y}_{\mathbf{1}}\right\} \cup \mathbf{Y}_{\mathbf{u}}\right) \backslash\left\{\mathbf{y}_{\mathbf{j}}\right\}\right)-\beta_{1}\left(\mathbf{Y}_{\mathbf{u}} \cup \tilde{\mathbf{Y}} \backslash\left\{\mathbf{y}_{\mathbf{j}}\right\}\right)=$ $7-4>\beta_{1}\left(\tilde{\mathbf{Y}} \cup\left\{\mathbf{y}_{\mathbf{1}}\right\} \cup \mathbf{Y}_{\mathbf{u}}\right)-\beta_{1}\left(\tilde{\mathbf{Y}} \cup \mathbf{Y}_{\mathbf{u}}\right)=8-7 \stackrel{\text { def }}{=} \widetilde{k_{1}}$. Let us search now a $\mathbf{y}_{\mathbf{1}}$-topped path $P$ which length is greater or equal to $\widetilde{k_{1}}+1=2$ and if possible includes edge $\left(\mathbf{x}_{\mathbf{2}}, \mathbf{x}_{1}\right)$. We can choose $P=\mathbf{x}_{2} \rightarrow \mathbf{x}_{1} \rightarrow \mathbf{y}_{1}$ with $\mathbf{v}_{\mathbf{P}}=\mathbf{x}_{2}$. Moreover, $S_{1}^{o}\left[\left\{\mathbf{x}_{\mathbf{2}}\right\}, \tilde{\mathbf{Y}} \cup\left\{\mathbf{y}_{\mathbf{2}}\right\}\right]=\left\{\mathbf{x}_{\mathbf{2}}\right\}$ and so, we verify that edge $\left(\mathbf{x}_{\mathbf{2}}, \mathbf{x}_{\mathbf{1}}\right)$ specific to the mode 1 belongs to a $S_{1}^{o}\left[\left\{\mathbf{x}_{\mathbf{2}}\right\}, \tilde{\mathbf{Y}} \cup\left\{\mathbf{y}_{\mathbf{2}}\right\}\right]-\tilde{\mathbf{Y}} \cup\left\{\mathbf{y}_{\mathbf{1}}\right\}$ path. Condition Cond4. is then satisfied. This graphic condition characterizes algebraic relation $\frac{\dot{y}_{1}}{\lambda_{9} \lambda_{13}}-\frac{\dot{y}_{2}}{\lambda_{5} \lambda_{14}}=0$ depending on $\lambda_{9}$ which is specific to mode 1 only. This relation is not satisfied for mode 2 because, in this case $\dot{y}_{1}$ is identically zero. Relation $\dot{y}_{1}=0$ is specific to mode 2 even if there is no specific coefficient to mode 2 which appears.

\subsection{Global observability analysis}

According to Definition 4, we can immediately state:

Proposition $3 \operatorname{SSLS}\left(\Sigma_{\Lambda}\right)$, with two possible modes $q \in$ $\{1,2\}$, represented by digraph $\mathcal{G}\left(\Sigma_{\Lambda}\right)$, is generically input and state observable iff all the following conditions hold: i.) $\left(\Sigma_{\Lambda}\right)$ is generically location observable i.e. one of the conditions of Proposition 2 is satisfied; ii.) $\forall q \in\{1,2\}, \theta_{q}[\mathbf{X} \cup \mathbf{U}, \mathbf{X} \cup \mathbf{Y}]=n+m$; iii.) $\forall q \in\{1,2\},\left\{\mathbf{x}_{\mathbf{i}} \in \mathbf{X} \mid \rho_{q}\left[\mathbf{U} \cup\left\{\mathbf{x}_{\mathbf{i}}\right\}, \mathbf{Y}\right]=\right.$ $\left.\rho_{q}[\mathbf{U}, \mathbf{Y}]\right\} \subseteq V_{e s s, q}[\mathbf{U}, \mathbf{Y}]$.

The conditions of Proposition 3 need few information about the system and they can be checked by means of well-known combinatorial techniques. From a computational point of view, the decomposition of the system into two subsystems which requires $n+p+1$ computations of maximal linking size and at most $n+q$ computations of maximal matching size. Using Ford-Fulkerson algorithm, the computation of the maximal linking size necessitates algorithms which have a complexity order $O\left(K^{2} \sqrt{M}\right)$, where $M$ is the number of edges in the digraph and $K=n+p+q$ the number of vertices. For our digraphs, in the worst case $M=(m+1) \cdot n^{2}+n \cdot p+n \cdot q+q \cdot p$ and so $O\left(K^{2} \sqrt{M}\right)=O\left(n^{3}\right)$.

The two first location observability conditions are equivalent to a computation of the successors of some state and input vertices in a digraph. This can be done using an algorithm of complexity order $O(M)$. The two last conditions of Proposition 2 necessitate first the computation of the $\beta$ function, for which we use an algorithm which complexity order equals $O\left(K^{3} \cdot M^{0.5}\right)=O\left(n^{4}\right)$ (Boukhobza et al., 2007). Otherwise, in order to avoid the combinatorial difficulties to find the corresponding outputs $\mathbf{y}_{\mathbf{i}}, \tilde{\mathbf{Y}}$ and $\mathbf{Y}_{\mathbf{u}}$, we must select first all the output vertices of $\mathbf{Y}_{\mathbf{1 , q}}$ to which there exist paths including $\mathcal{E}_{q}$ edges. It will necessitate, in the worst case $O\left(n^{2}\right)$ computations of function $\beta$. So, the two last conditions can be implemented using a polynomial complexity order algorithm $O\left(n^{6}\right)$. Condition ii.) and iii.) of Proposition 3 needs an algorithm of complexity order $O\left(n^{4}\right)$ (Boukhobza et al., 2007).

\subsection{Generalization to multi-mode case}

The main difficulty is to generalize location observability criterion and more precisely the matrix decomposition related to each mode into two parts: a common one and a specific one. Consider SSLS $\left(\Sigma_{\Lambda}\right)$, where $Q \stackrel{\text { def }}{=}\{1,2, \ldots, N\}$. We take the following notations: for $q \in\{1,2, \ldots, N\}, q^{\prime} \in$ $\{1,2, \ldots, N\}$ with $q \neq q^{\prime}, A^{\lambda}(q)=A_{q, q^{\prime}}^{\lambda}+A_{q, \overline{q^{\prime}}}^{\lambda}$, where $A_{q, q^{\prime}}^{\lambda}=A_{q^{\prime}, q}^{\lambda}$ denotes the common part between $A^{\lambda}(q)$ and $A^{\lambda}\left(q^{\prime}\right)$ and $A_{q, \overline{q^{\prime}}}^{\lambda}$ represent the specific part of $A^{\lambda}(q)$ relatively to $A^{\lambda}\left(q^{\prime}\right)$. Similarly, $B^{\lambda}(q)=B_{q, q^{\prime}}^{\lambda}+B_{q, \overline{q^{\prime}}}^{\lambda}$, $C^{\lambda}(q)=C_{q, q^{\prime}}^{\lambda}+C_{q, \overline{q^{\prime}}}^{\lambda}$ and $D^{\lambda}(q)=D_{q, q^{\prime}}^{\lambda}+D_{q, \overline{q^{\prime}}}^{\lambda}$. We have multiple decompositions for matrices $A^{\lambda}(q), B^{\lambda}(q), C^{\lambda}(q)$ and $D^{\lambda}(q)$ useful only in location observability study. To each of these matrices, we associate, as in Section 3, an edge subset. Thus, we state:

Proposition $4 \operatorname{SSLS}\left(\Sigma_{\Lambda}\right)$ associated to digraph $\mathcal{G}\left(\Sigma_{\Lambda}\right)$ is generically location observable iff for each pair $q \in$ $\{1,2, \ldots, N\}, q^{\prime} \in\{1,2, \ldots, N\}$ with $q \neq q^{\prime}$, Proposition 2 is satisfied by substituting edge subsets $\mathcal{E}_{0}$ by $A_{q, q^{\prime}}$-edges $\cup B_{q, q^{\prime}}$-edges $\cup C_{q, q^{\prime}}$-edges $\cup D_{q, q^{\prime}}$-edges, $\mathcal{E}_{1}$ by $A_{q, \overline{q^{\prime}}}$-edges $\cup B_{q, \overline{q^{\prime}}}$-edges $\cup C_{q, \overline{q^{\prime}}}$-edges $\cup D_{q, \overline{q^{\prime}}}$ edges and $\mathcal{E}_{2}$ by $A_{q^{\prime}, \bar{q}^{-}}$edges $\cup B_{q^{\prime}, \bar{q}^{-}}$-edges $\cup C_{q^{\prime}, \bar{q}^{-}}$-edges $\cup$ $D_{q^{\prime}, \bar{q}^{-}}$-edges. 


\section{Conclusion}

In this paper, we propose a graph-theoretic tool to analyze discrete and continuous state and input generic observability for switching structured linear systems with unknown inputs. This problem has not been solved previously in the literature. Our approach uses classical programming techniques and is free from numerical difficulties since it uses well-known combinatorial techniques which complexity is polynomial $\left(O\left(n^{6}\right)\right)$. This makes our approach well suited to large scale systems.

\section{References}

Babaali, M. \& Pappas, G. J., (2005). Observability of switched linear systems in continuous time. In M. Morari and L. Thiele, Editors, Hybrid Systems: Computation and Control, volume 3414 of Lecture Notes in Computer Science, 103-117. Springer Berlin / Heidelberg.

Bemporad, A., Ferrari-Trecate, G. \& Morari, M. (2000). bservability and controllability of piecewise affine and hybrid systems. IEEE Transactions on Automatic Control, 45(10), 1864-1876.

Boukhobza, T., Hamelin, F. \& Martinez-Martinez, S. (2007). State and input observability for structured linear systems: A graph-theoretic approach. Automatica, 43(7), 1204-1210.

Boukhobza, T., Hamelin, F., Martinez-Martinez, S. \& Sauter, D. (2009) Structural analysis of the partial state and input observability for structured linear systems. Application to distributed control systems. European Journal of Control, 15(5), 503-517.

Chaib, S., Boutat, D., Benali, A. \& Barbot, J-P (2005). bservability of the discrete state for dynamical piecewise hybrid systems. Nonlinear Analysis, 63(3), 423-438.

Collins, P. \& van Schuppen, J. H., (2004). Observability of piecewiseaffine hybrid systems. In R. Alur and G. J. Pappas, Editors, Hybrid Systems: Computation and Control, volume 2993 of Lecture Notes in Computer Science, 103-117. Springer Berlin / Heidelberg.

De Santis, E., Di Benedetto, M. D. \& Pola, G. (2006). Observability of internal variables in interconnected switching systems. Proceedings of the $45^{\text {th }}$ IEEE Conference on Decision and Control, 4121-4126, U.S.A.

De Santis, E., Di Benedetto, M. D. \& Pola, G. (2009). Observability and detectability of linear switching systems: A structural approach. arXiv:0802.4045v1 [math.DS]

Dion, J-M., Commault, C., \& Van der Woude, J. (2003). Generic properties and control of linear structured systems: A survey. Automatica, $39(7), 1125-1144$

Hovelaque, V., Commault, C. \& Dion, J-M. (1996). Analysis of linear structured systems using a primal-dual algorithm. System \& Control Letters, 27(2), 73-85.

Murota, K. (1987). System Analysis by Graphs and Matroids. SpringerVerlag. New York, U.S.A

Pina, L. \& Botto, A. (2007). Simultaneous state and input estimation of hybrid systems with unknown inputs. Automatica, 42(5),755-762.

Reinschke, K. J. (1988). Multivariable Control. A Graph Theoretic Approach. Springer-Verlag. New York, U.S.A.

Silverman, L. M. (1969). Inversion of multivariable linear systems. IEEE Transactions on Automatic Control, AC-14(3), 270-276.

Trentelman, H. L., Stoorvogel, A. A. \& Hautus, M. (2001). Control Theory for Linear Systems. Springer, London, U.K.

van der Woude, J. W. (2000). The generic number of invariant zeros of a structured linear system. SIAM Journal of Control and Optimization, $38(1), 1-21$.

J. W. van der Woude, C. Commault, and J. M. Dion, Zero orders and dimensions of some invariant subspaces in linear structured systems. Mathematics of Control, Signals and Systems, 16 (2-3), 225-237.

Vidal, R., Chiuso, A., Soatto, S. \& Sastry, S. (2003). Observability of linear hybrid systems. In O. Maler and A. Pnueli, Editors, Hybrid Systems: Computation and Control, volume 2623 of Lecture Notes in Computer Science, 526-539. Springer Berlin / Heidelberg.

$\mathrm{Vu}$, L. \& Liberzon, D. (2006). On invertibility of switched linear systems. Proceedings of the $45^{\text {th }}$ IEEE Conference on Decision and Control, 4081-4086, U.S.A. 